\title{
A Brouwer-like theorem for orientation reversing homeomorphisms of the sphere
}

\author{
by \\ Marc Bonino (Paris)
}

\begin{abstract}
We provide a topological proof that each orientation reversing homeomorphism of the 2 -sphere which has a point of period $k \geq 3$ also has a point of period 2 . Moreover if such a $k$-periodic point can be chosen arbitrarily close to an isolated fixed point $o$ then the same is true for the 2-periodic point. We also strengthen this result by proving that if an orientation reversing homeomorphism $h$ of the sphere has no 2-periodic point then the complement of the fixed point set can be covered by invariant open sets where $h$ is conjugate either to the map $(x, y) \mapsto(x+1,-y)$ or to the map $(x, y) \mapsto \frac{1}{2}(x,-y)$.
\end{abstract}

\section{INTRODUCTION}

A classical theorem of Brouwer asserts, in its weaker version, that an orientation preserving homeomorphism of the plane $\mathbb{R}^{2}$ which possesses a $k$-periodic point, $k \geq 2$, also has a fixed point (see [2] or [3], [7], [12]). The aim of this paper is to give a counterpart of this result in the framework of orientation reversing homeomorphisms. Considering homeomorphisms of the 2 -sphere $\mathbb{S}^{2}$, we first prove that if such a homeomorphism reverses the orientation and has a $k$-periodic point, $k \geq 3$, then it also admits a 2-periodic point (Theorem 3.1). Using Nielsen-Thurston theory, such a result was already proved for $C^{1}$-diffeomorphisms ([10]) and one could probably drop the smoothness assumption working again with this powerful theory. We give a topological proof based on the computation of the Lefschetz index on suitable open subsets of $\mathbb{S}^{2}$. This point of view emphasizes the analogy with the result of Brouwer mentioned above and leads, we hope, to a fairly intuitive proof. For example these arguments allow one to localize the 2-periodic orbit on both sides of a Jordan curve with some index properties, and can be readily adapted to give a local version of Theorem 3.1: if an isolated fixed point is the limit of $k$-periodic points $(k \geq 3)$ then it is also the limit of 2-periodic points (Theorem 4.3).

2000 Mathematics Subject Classification: 37E30, 37C25, 37Bxx. 
Section 5 is devoted to a "strong version" of our result. This is motivated by the Brouwer plane translation theorem which, roughly speaking, asserts that if $h$ is a fixed point free orientation preserving planar homeomorphism then every point is contained in a simply connected invariant domain where $h$ is conjugate to a translation (see [9], [12], [16] for modern references). It is then natural to expect a version of our result which would assert that an orientation reversing homeomorphism $h$ of $\mathbb{S}^{2}$ without a 2-periodic point has "obvious" dynamics on some invariant open sets covering the complement of the fixed point set $\operatorname{Fix}(h)$. This is carried out in Theorem 5.1 where such open sets are shown to exist, where $h$ is conjugate either to the map $(x, y) \mapsto(x+1,-y)$ or to the map $(x, y) \mapsto \frac{1}{2}(x,-y)$.

\section{BACKGROUND}

2.1. Notations and basic definitions. The plane $\mathbb{R}^{2}$ is endowed with its euclidean norm $\|\cdot\|$ and we think of the 2-sphere $\mathbb{S}^{2}$ as the one-point compactification of $\mathbb{R}^{2}$, that is, $\mathbb{S}^{2}=\mathbb{R}^{2} \cup\{\infty\}$. Thus a planar homeomorphism is identified with a homeomorphism of $\mathbb{S}^{2}$ fixing the point $\infty$ and our results are also valid for such a homeomorphism.

For $X \subset Y \subset \mathbb{S}^{2}$, we write respectively $\operatorname{Int}_{Y}(X), \mathrm{Cl}_{Y}(X)$ and $\partial_{Y}(X)=$ $\mathrm{Cl}_{Y}(X) \backslash \operatorname{Int}_{Y}(X)$ for the interior, closure and frontier of $X$ with respect to $Y$. For simplicity we omit the subscript $Y$ when $Y=\mathbb{S}^{2}$. We also denote by $\pi_{0}(X)$ the set of all connected components of $X$.

An arc is a subset of $\mathbb{S}^{2}$ homeomorphic to the interval $[0,1]$, and an open arc is an arc with its two endpoints removed. If $\gamma$ is an arc provided with an orientation and $a, b$ two points met in this order on $\gamma$, then $[a, b]_{\gamma}$ is the subarc from $a$ to $b$ for the chosen orientation of $\gamma$.

A topological closed disc is a subset of $\mathbb{S}^{2}$ homeomorphic to the closed unit disc.

For any map $f: E \rightarrow F$, the fixed point set $\{z \in E \mid f(z)=z\}$ is denoted by $\operatorname{Fix}(f)$. A point $z \in E$ is said to be a $k$-periodic point of $f$ if $k$ is the smallest positive integer such that the sequence $z, f(z), \ldots, f^{k}(z)$ is well defined and $f^{k}(z)=z$.

2.2. Jordan curves and Jordan domains. A Jordan curve is a subset of $\mathbb{S}^{2}$ homeomorphic to the unit circle $\mathbb{S}^{1}$. According to the Jordan Theorem, the complement $\mathbb{S}^{2} \backslash J$ of a Jordan curve $J$ has exactly two connected components and $J$ is their common frontier. An open subset of $\mathbb{S}^{2}$ which is a connected component of the complement of a Jordan curve is said to be a Jordan domain. If $J$ is a Jordan curve with a given orientation and if $a \neq b$ are two points of $J$, then $[a, b]_{J}$ denotes the arc on $J$ from $a$ to $b$ for 
this orientation of $J$. Note that our vocabulary slightly differs from the one usually used in the literature, where a Jordan curve $J$ is often defined as a subset of $\mathbb{R}^{2}$ and a Jordan domain as the bounded component of $\mathbb{R}^{2} \backslash J$.

For later use, we now collect a few propositions about Jordan domains. The first one is a straightforward adaptation of a result of Kerékjártó:

Proposition 2.1. Let $U, U^{\prime}$ be two Jordan domains such that $U \cap U^{\prime} \neq \emptyset$ and $\left(\mathbb{S}^{2} \backslash \mathrm{Cl}(U)\right) \cap\left(\mathbb{S}^{2} \backslash \mathrm{Cl}\left(U^{\prime}\right)\right) \neq \emptyset$. Then every connected component of $U \cap U^{\prime}$ is also a Jordan domain, whose frontier is contained in $\partial U \cup \partial U^{\prime}$.

Indeed, if we assume that $\mathrm{Cl}(U)$ and $\mathrm{Cl}\left(U^{\prime}\right)$ are contained in the plane $\mathbb{R}^{2}$ then the hypothesis $\left(\mathbb{S}^{2} \backslash \mathrm{Cl}(U)\right) \cap\left(\mathbb{S}^{2} \backslash \mathrm{Cl}\left(U^{\prime}\right)\right) \neq \emptyset$ is of course satisfied and Proposition 2.1 is a well known result of Kerékjártó ([14]). In the general case, choose a point $z \in\left(\mathbb{S}^{2} \backslash \mathrm{Cl}(U)\right) \cap\left(\mathbb{S}^{2} \backslash \mathrm{Cl}\left(U^{\prime}\right)\right)$ and a homeomorphism $\varphi$ of $\mathbb{S}^{2}$ such that $\varphi(z)=\infty$. We reduce to the previous situation by considering $\mathrm{Cl}(\varphi(U))=\varphi(\mathrm{Cl}(U))$ and $\mathrm{Cl}\left(\varphi\left(U^{\prime}\right)\right)=\varphi\left(\mathrm{Cl}\left(U^{\prime}\right)\right)$.

We also have:

Lemma 2.2. Let $U, V$ be two Jordan domains such that $V \subset U, V \neq U$, and $\partial V \cap \partial U$ contains at least two points.

(1) For any $\mu \in \pi_{0}(U \cap \partial V)$ we have:

(i) $\mu$ is an open arc lying in $U$ with its two endpoints $x=x(\mu)$, $y=y(\mu)$ in $\partial U$ (it is usually said that $\mu$ is a cross-cut of $U$ ).

(ii) We have a partition $U \backslash \mu=U_{\mu}^{\prime} \cup U_{\mu}^{\prime \prime}$, where $U_{\mu}^{\prime}$ (resp. $\left.U_{\mu}^{\prime \prime}\right)$ is the Jordan domain contained in $U$ whose frontier is $\mu \cup[x, y]_{\partial U}$ (resp. $\left.\mu \cup[y, x]_{\partial U}\right)$.

(iii) The Jordan domain $V$ is contained either in $U_{\mu}^{\prime}$ or in $U_{\mu}^{\prime \prime}$.

Notation: We write $U_{\mu, V}$ for the connected component of $U \backslash \mu$ containing $V$ and $\mu_{*}$ for the arc in $\partial U$ with endpoints $x, y$ such that $\mu \cup \mu_{*}=\partial U_{\mu, V}$.

(2) If $a \in U \backslash \mathrm{Cl}(V)$, then there exists a unique $\mu=\mu(a) \in \pi_{0}(U \cap \partial V)$ such that $a \notin U_{\mu, V}$ (see Fig. 1).

Proof. (1) (i) follows from the fact that $U \cap \partial V=\partial V \backslash \partial U$ is not connected. (ii) is a classical result of plane topology (known as the $\Theta$-Curve Lemma). It can be proved with elementary arguments (see for example [18, Theorem 11.8, p. 119]). It can also be obtained as a corollary of the Schoenflies Theorem, giving a homeomorphism of $\mathbb{S}^{2}$ mapping respectively $\partial U$ and $\mu$ onto $\mathbb{S}^{1}$ and $(-1,1) \times\{0\}$. For (iii), it is enough to remark that $V$ is a connected subset of $U \backslash \mu$.

(2) Choose $a^{\prime}$ to be any point in $V$. Since $a, a^{\prime}$ are separated in $\mathbb{S}^{2}$ by the closed set $\partial V$ they are also separated in $U$ by a connected component $\mu$ 


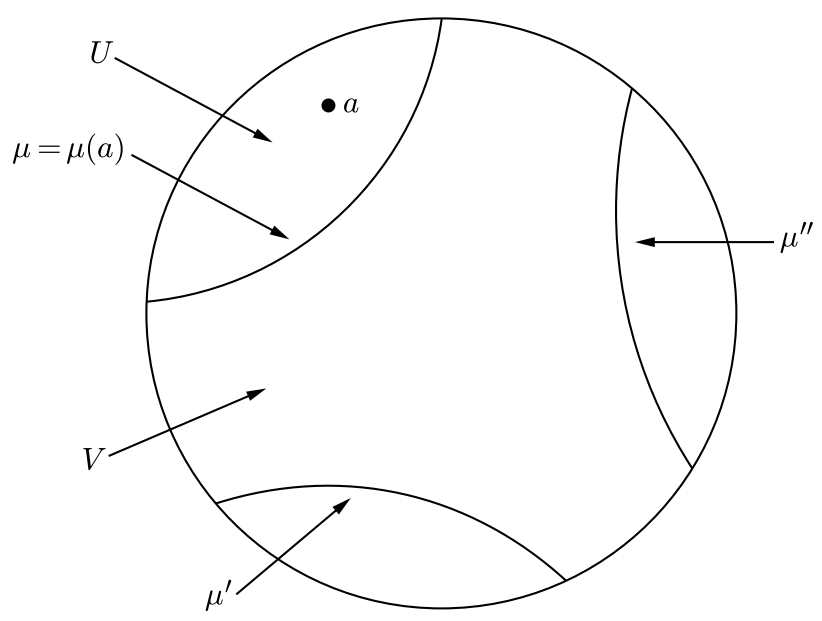

Fig. 1. The Jordan domains $U, V$ and the $\operatorname{arcs} \mu$

of $U \cap \partial V$ (see [18, Theorem 7.1, p. 151]) and we then have $a^{\prime} \in V \subset U_{\mu, V}$, $a \notin U_{\mu, V}$. Now suppose that we can find two connected components $\mu \neq \nu$ of $U \cap \partial V$ such that $a \notin U_{\mu, V} \cup U_{\nu, V}$ and consider the partitions

$$
U \backslash \mu=U_{\mu}^{\prime} \cup U_{\mu}^{\prime \prime}, \quad U \backslash \nu=U_{\nu}^{\prime} \cup U_{\nu}^{\prime \prime}
$$

given by (1)(ii). Assume for example that $U_{\mu}^{\prime}=U_{\mu, V}$ and $U_{\nu}^{\prime}=U_{\nu, V}$. Since $\mu \cap \nu=\emptyset$, we have either $\mu \subset U_{\nu, V}$ or $\mu \subset U_{\nu}^{\prime \prime}$. The latter case is actually impossible since $\mu \subset \mathrm{Cl}(V)$ and $U_{\nu}^{\prime \prime} \cap V=\emptyset$. It follows that

$$
a \in U_{\nu}^{\prime \prime} \subset U \backslash U_{\nu, V} \subset U \backslash \mu \text { and consequently } U_{\nu}^{\prime \prime} \subset U_{\mu}^{\prime \prime} .
$$

Reversing the roles of $\nu$ and $\mu$ we obtain $U_{\mu}^{\prime \prime} \subset U_{\nu}^{\prime \prime}$ so $U_{\mu}^{\prime \prime}=U_{\nu}^{\prime \prime}$ and finally

$$
\mu=U \cap \partial U_{\mu}^{\prime \prime}=U \cap \partial U_{\nu}^{\prime \prime}=\nu,
$$

a contradiction.

2.3. Lefschetz index. Let $M$ be a manifold (more generally, a Euclidean neighbourhood retract), $U$ an open subset of $M$ and $\varphi: U \rightarrow M$ a continuous map such that $\operatorname{Fix}(\varphi)$ is compact. One can define the fixed point index, or Lefschetz index, $\mathrm{I}(\varphi) \in \mathbb{Z}$ (see [5]) with the following properties:

Properties 2.3. (1) $\mathrm{I}(\varphi)$ depends only on the set $\operatorname{Fix}(\varphi)$, that is, $\mathrm{I}(\varphi)$ $=\mathrm{I}\left(\left.\varphi\right|_{U^{\prime}}\right)$ for any open set $U^{\prime}$ such that $\operatorname{Fix}(\varphi) \subset U^{\prime} \subset U$.

(2) If $\operatorname{Fix}(\varphi)=\emptyset$ then $I(\varphi)=0$.

(3) Additivity: If $U=\bigcup_{i=1}^{n} U_{i}$, where $U_{1}, \ldots, U_{n}$ are open, and if the sets $\operatorname{Fix}\left(\left.\varphi\right|_{U_{i}}\right)$ are compact and pairwise disjoint $(1 \leq i \leq n)$ then

$$
\mathrm{I}(\varphi)=\sum_{i=1}^{n} \mathrm{I}\left(\left.\varphi\right|_{U_{i}}\right) .
$$


(4) Homotopy Invariance: If $\left(\varphi_{t}: U \rightarrow M\right)_{0 \leq t \leq 1}$ is a homotopy from $\varphi_{0}=\varphi$ to $\varphi_{1}$ such that $\bigcup_{0 \leq t \leq 1} \operatorname{Fix}\left(\varphi_{t}\right)$ is compact, then $\mathrm{I}(\varphi)=\mathrm{I}\left(\varphi_{1}\right)$.

(5) Topological Invariance: If $\bar{\psi}: M \rightarrow M$ is a homeomorphism then the maps $\varphi$ and $\left.\psi \circ \varphi \circ\left(\psi^{-1}\right)\right|_{\psi(U)}: \psi(U) \rightarrow M$ have the same Lefschetz index.

Proof. The first four properties are stated in [5] so we just give the argument for the fifth one. It is in fact a consequence of the following Commutativity Property of the Lefschetz index (see [5]):

Let $U_{i}$ be an open set of a manifold $M_{i}(i \in\{1,2\})$ and let $k_{1}: U_{1} \rightarrow M_{2}$, $k_{2}: U_{2} \rightarrow M_{1}$ be two continuous maps. Then the composite maps

$$
\begin{array}{ll}
k_{1}^{-1}\left(U_{2}\right) \rightarrow M_{1}, & x \mapsto k_{2}\left(k_{1}(x)\right), \\
k_{2}^{-1}\left(U_{1}\right) \rightarrow M_{2}, & x \mapsto k_{1}\left(k_{2}(x)\right),
\end{array}
$$

have homeomorphic fixed point sets. They also have the same Lefschetz index if their fixed point sets are compact.

We remark that $\operatorname{Fix}\left(\left.\psi \circ \varphi \circ\left(\psi^{-1}\right)\right|_{\psi(U)}\right)=\psi(\operatorname{Fix}(\varphi))$ is compact, which ensures directly that the Lefschetz index of $\left.\psi \circ \varphi \circ\left(\psi^{-1}\right)\right|_{\psi(U)}$ is defined. Now use the Commutativity Property with

$$
k_{1}=\left.\left(\psi^{-1}\right)\right|_{\psi(U)}: \psi(U) \rightarrow M, \quad k_{2}=\psi \circ \varphi: U \rightarrow M
$$

to obtain

$$
\mathrm{I}\left(\left.\psi \circ \varphi \circ\left(\psi^{-1}\right)\right|_{\psi(U)}\right)=\mathrm{I}\left(\left.\varphi\right|_{\varphi^{-1}(U)}\right) .
$$

Furthermore we have obviously

$$
\operatorname{Fix}(\varphi) \subset \varphi^{-1}(U) \subset U
$$

and we conclude by applying Property 2.3(1).

Notations. We will deal with continuous maps $f: \mathcal{U} \rightarrow \mathbb{S}^{2}$, where $\mathcal{U} \subset \mathbb{S}^{2}$ is a given open set $\left(\mathcal{U}=\mathbb{S}^{2}\right.$ except in Section 4$)$, and we will calculate the Lefschetz index of $\varphi=\left.f\right|_{U}$ for various open sets $U \subset \mathcal{U}$. Thus we will speak of the index of $f$ on $U$ and write $\operatorname{Ind}(f, U)$ instead of $\mathrm{I}\left(\left.f\right|_{U}\right)$. Moreover, if $U$ contains exactly one fixed point $z$ of $f$, we recall that $\operatorname{Ind}(f, U)$ is said to be the Lefschetz index of $z$ (for the map $f$ ) and is also denoted $\operatorname{Ind}(f, z)$.

The following lemma follows from Properties 2.3.

Lemma 2.4. Let $U_{1}, U_{2}$ be two open subsets of $\mathbb{S}^{2}$ and let $f: \mathbb{S}^{2} \rightarrow \mathbb{S}^{2}$ be a continuous map. Suppose that $U_{i} \cap \operatorname{Fix}(f)$ is compact $(i \in\{1,2\})$. Then there are only finitely many connected components $V$ of $U_{1} \cap U_{2}$ such that $\operatorname{Ind}(f, V) \neq 0$, and

$$
\operatorname{Ind}\left(f, U_{1} \cap U_{2}\right)=\sum_{V \in \pi_{0}\left(U_{1} \cap U_{2}\right)} \operatorname{Ind}(f, V) .
$$


Proof. We can suppose $U_{1} \cap U_{2} \neq \emptyset$. The set $U_{1} \cap U_{2} \cap \operatorname{Fix}(f)$ is compact so there exists a finite open covering

$$
U_{1} \cap U_{2} \cap \operatorname{Fix}(f) \subset V_{1} \cup \cdots \cup V_{n}
$$

where $V_{1}, \ldots, V_{n}$ are some connected components of $U_{1} \cap U_{2}$. According to Properties 2.3(1)-(2) we have $\operatorname{Ind}\left(f, U_{1} \cap U_{2}\right)=\operatorname{Ind}\left(f, V_{1} \cup \cdots \cup V_{n}\right)$ and $\operatorname{Ind}(f, V)=0$ for any $V \in \pi_{0}\left(U_{1} \cap U_{2}\right) \backslash\left\{V_{1}, \ldots, V_{n}\right\}$. Then by Property $2.3(3)$ we obtain

$$
\operatorname{Ind}\left(f, U_{1} \cap U_{2}\right)=\sum_{i=1}^{n} \operatorname{Ind}\left(f, V_{i}\right)=\sum_{V \in \pi_{0}\left(U_{1} \cap U_{2}\right)} \operatorname{Ind}(f, V) .
$$

Although this is not essential in this paper, recall that, for planar maps, there is an intuitive interpretation for the Lefschetz index on Jordan domains:

Proposition 2.5. Let $\mathcal{U}$ be an open subset of $\mathbb{R}^{2}$ and let $f: \mathcal{U} \rightarrow \mathbb{R}^{2}$ be a continuous map. If $U$ is a Jordan domain such that $\mathrm{Cl}(U) \subset \mathcal{U}$ and $\partial U \cap \operatorname{Fix}(f)=\emptyset$ then $\operatorname{Ind}(f, U)$ is the degree of the map

$$
\mathbb{S}^{1} \rightarrow \mathbb{S}^{1}, \quad t \mapsto \frac{f(u(t))-u(t)}{\|f(u(t))-u(t)\|},
$$

where $u: \mathbb{S}^{1} \rightarrow \partial U=u\left(\mathbb{S}^{1}\right)$ is any homeomorphism which endows $\partial U$ with its counterclockwise orientation.

This result is for example a consequence of [5, exercise 5, p. 207]. In other words, if $f$ and $U$ are as in Proposition 2.5 then $\operatorname{Ind}(f, U)$ is the winding number of the vector $f(z)-z$ when $z$ moves along the Jordan curve $\partial U$ in the counterclockwise direction. For this reason $\operatorname{Ind}(f, U)$ is also said to be the index of $f$ on the curve $\partial U$ and is often denoted $\operatorname{Ind}(f, \partial U)$ instead of $\operatorname{Ind}(f, U)$ in the literature.

We end this section with an index zero lemma which will be repeatedly used in this paper:

Lemma 2.6. Let $U, V$ be two Jordan domains such that $V \subset U, V \neq U$, and $\partial V \cap \partial U$ contains at least two points, and let $f: \mathbb{S}^{2} \rightarrow \mathbb{S}^{2}$ be a continuous map. Assume furthermore that

(i) $f$ has no fixed point in $\partial V$,

(ii) $U \cap \partial V \cap f(U)=\emptyset$,

(iii) there exists $\mu \in \pi_{0}(U \cap \partial V)$ such that, in the notations of Lemma 2.2, $f\left(\mu_{*}\right) \cap U=\emptyset$.

Then $\operatorname{Ind}(f, V)=0$.

Proof. Because of (i), $\operatorname{Ind}(f, V)$ is defined. We consider the Jordan domain $U_{\mu, V}$ and the arc $\mu_{*}$ associated to $\mu$, as explained in Lemma 2.2. Since 
$\partial U_{\mu, V}=\mu \cup \mu_{*}$ it is easy to construct a homotopy

$$
\mathrm{Cl}\left(U_{\mu, V}\right) \times[0,1] \rightarrow \mathrm{Cl}\left(U_{\mu, V}\right), \quad(z, t) \mapsto r_{t}(z),
$$

with the following properties:

1. $r_{0}$ is the identity map of $C l\left(U_{\mu, V}\right)$,

2. $r_{1}\left(C l\left(U_{\mu, V}\right)\right)=\mu_{*}$,

3. $\forall t \in[0,1] \forall z \in \mu_{*} r_{t}(z)=z$,

4. if $0<t \leq 1$ then $r_{t}\left(\mathrm{Cl}\left(U_{\mu, V}\right)\right) \subset U_{\mu, V} \cup \mu_{*}$.

Essentially, this simply means that $\left(r_{t}\right)_{0 \leq t \leq 1}$ is a strong deformation retraction of $\mathrm{Cl}\left(U_{\mu, V}\right)$ onto $\mu_{*}$. The additional fourth property ensures that the maps $f \circ r_{t}$ have no fixed point on $\partial V(0 \leq t \leq 1)$. Indeed, there is nothing to prove for $\left.f \circ r_{0}\right|_{\partial V}=\left.f\right|_{\partial V}$, and for $0<t \leq 1$ and $z \in \partial V \subset \mathrm{Cl}\left(U_{\mu, V}\right)$, we have:

- If $z \in \mu_{*}$ then $f \circ r_{t}(z)=f(z) \neq z$.

- If $z \in U_{\mu, V} \cup \mu$ then by (4),

$$
f \circ r_{t}(z) \in f\left(U_{\mu, V}\right) \cup f\left(\mu_{*}\right)
$$

and consequently $z \neq f \circ r_{t}(z)$ since, in view of (ii) and (iii),

$$
\partial V \cap\left(U_{\mu, V} \cup \mu\right) \cap f\left(U_{\mu, V}\right) \subset \partial V \cap U \cap f(U)=\emptyset
$$

and

$$
\partial V \cap\left(U_{\mu, V} \cup \mu\right) \cap f\left(\mu_{*}\right) \subset U \cap f\left(\mu_{*}\right)=\emptyset .
$$

Moreover we have

$$
f \circ r_{1}(V) \cap V \subset f \circ r_{1}\left(\mathrm{Cl}\left(U_{\mu, V}\right)\right) \cap U=f\left(\mu_{*}\right) \cap U=\emptyset,
$$

which gives $\operatorname{Ind}\left(f \circ r_{1}, V\right)=0$. The result then follows from Property 2.3(4) by considering the homotopy $\left(\left.f \circ r_{t}\right|_{V}\right)_{0 \leq t \leq 1}$.

\subsection{Translation arcs}

Definition 2.7. Let $f$ be a homeomorphism of $\mathbb{S}^{2}$. An $\operatorname{arc} \gamma$ is said to be a translation arc for $f$ if

1. one of its two endpoints, say $p$, is mapped by $f$ to the other one,

2. $\gamma \cap f(\gamma)=\{p, f(p)\} \cap\left\{f(p), f^{2}(p)\right\}$.

Note that, with the above definition, $\operatorname{Fix}(f)$ is necessarily disjoint from $\bigcup_{k \in \mathbb{Z}} f^{k}(\gamma)$. For convenience we also make the following convention. If $f$ is a given homeomorphism of $\mathbb{S}^{2}$ and $\gamma$ a translation arc for $f$ with endpoints $p$ and $f(p)$ then the $\operatorname{arcs} f^{k}(\gamma)$ are oriented from $f^{k}(p)$ to $f^{k+1}(p)(k \in \mathbb{Z})$. Of course $\gamma$ could also be thought of as a translation arc for $f^{-1}$ and the $\operatorname{arcs} f^{k}(\gamma)$ would then be oriented from $f^{k+1}(p)$ to $f^{k}(p)$. 
LEMMA 2.8. Let $h$ be a homeomorphism of $\mathbb{S}^{2}$ such that $h^{2} \neq \operatorname{Id}_{\mathbb{S}^{2}}$ and let $m$ be a point in $\mathbb{S}^{2} \backslash \operatorname{Fix}\left(h^{2}\right)$. Then at least one of the following two assertions holds:

(A1) There exists a translation arc $\alpha$ for $h$, with endpoints $p$ and $h(p)$, such that $\alpha \cap h(\alpha)=\{h(p)\}, \alpha \cap h^{2}(\alpha)=\{p\} \cap\left\{h^{3}(p)\right\}$ and $m \in$ $\alpha \backslash\{p, h(p)\}$.

(A2) There exists a translation arc $\beta$ for $h^{2}$, with endpoints $q$ and $h^{2}(q)$, such that $\beta \cap h(\beta)=\emptyset$ and $m \in \beta \backslash\left\{q, h^{2}(q)\right\}$.

Proof. Let $U$ be the connected component of $\mathbb{S}^{2} \backslash \operatorname{Fix}\left(h^{2}\right)$ which contains $m$. We know that $h^{2}(U)=U$ (see [4]), and since an open subset of $\mathbb{S}^{2}$ is arcwise connected, there exists an arc $\gamma$ lying in $U$ with endpoints $m$ and $h^{2}(m)$. We can slightly enlarge $\gamma$ to obtain a topological closed disc $\Delta$ such that $\gamma \subset \operatorname{Int}(\Delta) \subset \Delta \subset U$. For any $R>0$, denote by $D_{R}$ the closed disc in $\mathbb{R}^{2}$ with center $o=(0,0)$ and radius $R$. Up to conjugacy in $\mathbb{S}^{2}$, we can suppose that $m=o$ and $\Delta=D_{1}$. Define $R_{1}, R_{2}>0$ to be the unique real numbers such that

$$
\begin{gathered}
\partial D_{R_{1}} \cap h\left(\partial D_{R_{1}}\right)=D_{R_{1}} \cap h\left(D_{R_{1}}\right) \neq \emptyset, \\
\partial D_{R_{2}} \cap h^{2}\left(\partial D_{R_{2}}\right)=D_{R_{2}} \cap h^{2}\left(D_{R_{2}}\right) \neq \emptyset .
\end{gathered}
$$

Observe that, since $h^{2}(o) \in \operatorname{Int}\left(D_{1}\right) \cap h^{2}\left(\operatorname{Int}\left(D_{1}\right)\right)$, we necessarily have $R_{2}<1$ so

$$
D_{R_{2}} \subset D_{1} \subset \mathbb{S}^{2} \backslash \operatorname{Fix}\left(h^{2}\right) \subset \mathbb{S}^{2} \backslash \operatorname{Fix}(h) .
$$

Lemma 2.8 then follows from the comparison of $R_{1}$ and $R_{2}$ :

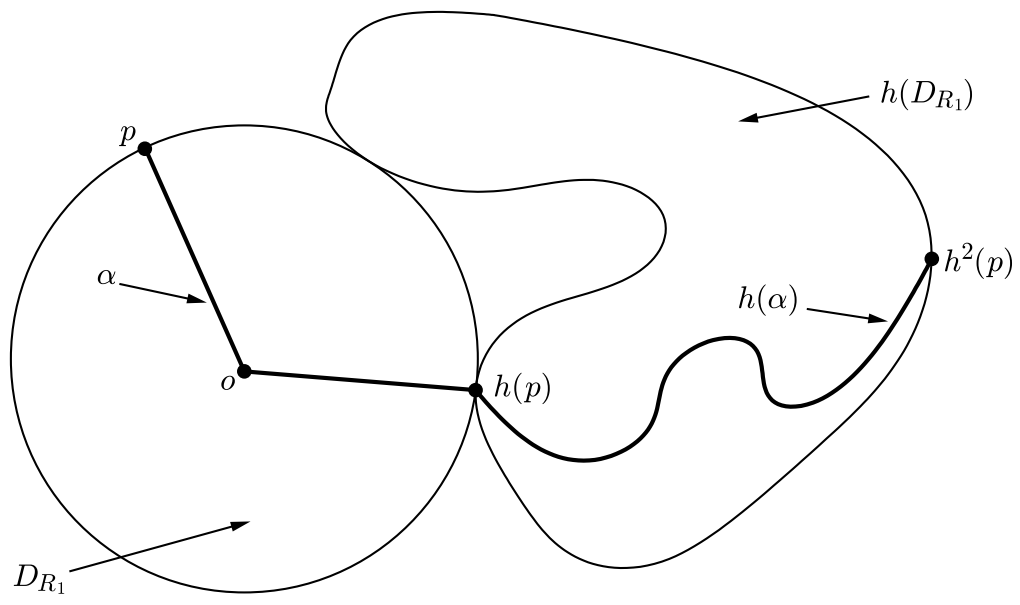

Fig. 2. The translation $\operatorname{arc} \alpha$

- If $R_{1} \leq R_{2}$, choose $p \in \partial D_{R_{1}}$ such that $h(p) \in \partial D_{R_{1}}$. Since $D_{R_{1}} \subset D_{R_{2}}$, the points $p, h(p), h^{2}(p)$ are distinct and any arc $\alpha$ from $p$ to $h(p)$ satisfying 
$o \in \alpha \backslash\{p, h(p)\} \subset \operatorname{Int}\left(D_{R_{1}}\right)$ (see Fig. 2) has the properties required in assertion (A1).

- If $R_{1}>R_{2}$, let $q \in \partial D_{R_{2}}$ be such that $h^{2}(q) \in \partial D_{R_{2}}$. Choose an arc $\beta$ from $q$ to $h^{2}(q) \neq q$ such that $o \in \beta \backslash\left\{q, h^{2}(q)\right\} \subset \operatorname{Int}\left(D_{R_{2}}\right)$. It is clear that $\beta$ is an arc as described in assertion (A2) (possibly with $q=h^{4}(q)$ ).

2.5. Brouwer's lemma. Let $f$ be an orientation preserving homeomorphism of $\mathbb{S}^{2}$. Suppose that $\gamma$ is a translation arc for $f$, with endpoints $p, f(p)$, such that $\bigcup_{k \in \mathbb{Z}} f^{k}(\gamma)$ is not a simple curve, i.e. the set $\{k \geq 1 \mid$ $\left.(\gamma \backslash\{f(p)\}) \cap f^{k}(\gamma \backslash\{f(p)\}) \neq \emptyset\right\}$ is nonempty. If $n$ denotes the minimum of the last set and $x$ the first point on $f^{n}(\gamma)$ to fall into $\gamma$, then

$$
C=[x, f(p)]_{\gamma} \cup \bigcup_{i=1}^{n-1} f^{i}(\gamma) \cup\left[f^{n}(p), x\right]_{f^{n}(\gamma)}
$$

is clearly a Jordan curve and we have:

Proposition 2.9 (Brouwer's lemma). Let $U$ be a connected component of $\mathbb{S}^{2} \backslash C$. Then $\operatorname{Ind}(f, U)=1$. In particular $f$ admits a fixed point in $U$.

Usually Brouwer's lemma ([3], [12, Appendice]) is stated for an orientation preserving homeomorphism $f$ of $\mathbb{R}^{2}$. The $\operatorname{arcs} f^{i}(\gamma)$ then lie in $\mathbb{R}^{2}$ and it is shown that $\operatorname{Ind}(f, U)=1$ for the bounded connected component $U$ of $\mathbb{R}^{2} \backslash C$. Proposition 2.9 is only a minor adaptation of that statement. Indeed, let $U_{1}$ and $U_{2}$ be the two connected components of $\mathbb{S}^{2} \backslash C$. According to the Lefschetz-Hopf Theorem (see for example [5]) we have

$$
2=\operatorname{Ind}\left(f, \mathbb{S}^{2}\right)=\operatorname{Ind}\left(f, U_{1}\right)+\operatorname{Ind}\left(f, U_{2}\right) .
$$

Consequently, $f$ admits at least one fixed point $z \in \mathbb{S}^{2} \backslash C$, say $z \in U_{1}$, and it is enough to check $\operatorname{Ind}\left(f, U_{2}\right)=1$. Choosing a homeomorphism $\varphi$ of $\mathbb{S}^{2}$ such that $\varphi(z)=\infty$ it is clear that $\varphi \circ f \circ \varphi^{-1}$ is a planar homeomorphism with $\varphi(\gamma)$ as a translation arc, hence the above references give $\operatorname{Ind}(\varphi \circ f$ $\left.\circ \varphi^{-1}, \varphi\left(U_{2}\right)\right)=1$. We derive $\operatorname{Ind}\left(f, U_{2}\right)=1$ from Property $2.3(5)$.

\section{FIRST RESULT: PERIOD $k \geq 3$ IMPLIES PERIOD 2}

In this section we prove

THEOREM 3.1. Let $h$ be an orientation reversing homeomorphism of the sphere $\mathbb{S}^{2}$ with a point of period at least three. Then $h$ also admits a 2periodic point. More precisely, there exist a Jordan curve $C \subset \mathbb{S}^{2} \backslash \operatorname{Fix}\left(h^{2}\right)$ and a point $z$ such that, writing $U, U^{\prime}$ for the two connected components of $\mathbb{S}^{2} \backslash C$, we have

$$
z=h^{2}(z) \in U, \quad h(z) \in U^{\prime}, \quad \operatorname{Ind}(h, U)=0, \quad \operatorname{Ind}\left(h^{2}, U\right)=1 .
$$


We introduce the following notation in order to avoid unpleasant repetitions.

Notation. $f, g$ being two homeomorphisms of $\mathbb{S}^{2}$, we write $f \sim g$ if and only if

1. $\forall i \in\{1,2\} \operatorname{Fix}\left(f^{i}\right)=\operatorname{Fix}\left(g^{i}\right)$ and $\operatorname{Ind}\left(f^{i}, \Omega\right)=\operatorname{Ind}\left(g^{i}, \Omega\right)$ for any open set $\Omega \subset \mathbb{S}^{2}$ such that $\Omega \cap \operatorname{Fix}\left(f^{i}\right)$ is compact,

2. $\forall z \in \operatorname{Fix}\left(f^{2}\right) \quad f(z)=g(z)$.

Clearly, $\sim$ defines an equivalence relation and Theorem 3.1 will be proved if its conclusion holds for a homeomorphism $g \sim h$.

Let us explain the main idea to detect the 2-periodic point $z \in U$. We will actually prove the following stronger result (although less meaningful from the dynamical viewpoint).

AN ADDITIONAL INDEX PROPERTY. There exists a connected component $U$ of $\mathbb{S}^{2} \backslash C$ such that, in addition to the above index properties, we have $\operatorname{Ind}\left(h^{2}, U \cap h(U)\right)=0$ (possibly with $U \cap h(U)=\emptyset$ ).

In particular this will show $\operatorname{Ind}\left(h^{2}, U\right) \neq \operatorname{Ind}\left(h^{2}, U \cap h(U)\right)$, and Property 2.3(1) then gives $\operatorname{Fix}\left(h^{2}\right) \cap U \neq \operatorname{Fix}\left(h^{2}\right) \cap U \cap h(U)$. In other words, there exists a point $z \in U$ such that $h^{2}(z)=z$ and $h(z)=h^{-1}(z) \in U^{\prime}=$ $\mathbb{S}^{2} \backslash \mathrm{Cl}(U)$, as announced in Theorem 3.1.

Finally, we remark that there is no loss in proving this last index property only for a homeomorphism $g \sim h$ :

Claim. Suppose $g \sim h$ and let $\Omega \subset \mathbb{S}^{2}$ be any open set. Then the indices $\operatorname{Ind}\left(g^{2}, \Omega \cap g(\Omega)\right)$ and $\operatorname{Ind}\left(h^{2}, \Omega \cap h(\Omega)\right)$ are simultaneously defined or not, and if defined, they are equal.

Indeed, $g$ and $h$ have exactly the same fixed points and the same 2periodic orbits so

$$
\begin{aligned}
\operatorname{Fix}\left(g^{2}\right) \cap \Omega \cap g(\Omega) & =\operatorname{Fix}\left(g^{2}\right) \cap \Omega \cap g(\Omega) \cap h(\Omega) \\
& =\operatorname{Fix}\left(h^{2}\right) \cap \Omega \cap g(\Omega) \cap h(\Omega) \\
& =\operatorname{Fix}\left(h^{2}\right) \cap \Omega \cap h(\Omega) .
\end{aligned}
$$

If these sets are compact we let $\Omega^{\prime}=\Omega \cap g(\Omega) \cap h(\Omega)$. It follows from Property 2.3(1) and from the definition of $\sim$ that

$$
\operatorname{Ind}\left(g^{2}, \Omega \cap g(\Omega)\right)=\operatorname{Ind}\left(g^{2}, \Omega^{\prime}\right)=\operatorname{Ind}\left(h^{2}, \Omega^{\prime}\right)=\operatorname{Ind}\left(h^{2}, \Omega \cap h(\Omega)\right) .
$$

\subsection{A proposition about translation arcs of $h$}

Proposition 3.2. Let $h$ be an orientation reversing homeomorphism of $\mathbb{S}^{2}$. Assume that we can find a translation arc $\alpha$ for $h$, with endpoints $p, h(p)$, such that: 
- $\alpha \cap h(\alpha)=\{h(p)\}, \alpha \cap h^{2}(\alpha)=\{p\} \cap\left\{h^{3}(p)\right\}$,

- $\alpha \cap h^{k}(\alpha) \neq \emptyset$ for an integer $k \geq 2$, i.e. the set $\bigcup_{k \in \mathbb{Z}} h^{k}(\alpha)$ is not a simple curve.

Then there exist a Jordan curve $C$ and a point $z$ as announced in Theorem 3.1 .

This proposition is a consequence of the three lemmas below. The first one allows us to reduce to the situation where, for a smallest $n \geq 2$, the iterate $h^{n}(\alpha)$ meets the arc $\alpha$ "in a nice way". This will be convenient to compute some indices on a suitable Jordan domain. We use the same technique as in the proof of [3, Theorem 1], observing that the "perturbations" of $h$ can be constructed without altering not only the fixed point set but also the set of 2-periodic orbits. For completeness and because similar lemmas will be used later in this paper, we give a rather detailed proof.

Lemma 3.3. Let $h$ and $\alpha$ be as in Proposition 3.2. Define $n$ to be the minimum of the set $\left\{k \geq 2 \mid \alpha \cap h^{k}(\alpha) \neq \emptyset\right\}$ and $x$ to be the first point on $h^{n}(\alpha)$ to fall into $\alpha$. Then there exists an orientation reversing homeomorphism $h_{*} \sim h$ admitting $\alpha_{*}=[x, h(p)]_{\alpha}$ as a translation arc such that $h_{*}(x)=h(p)$ and

- $\forall i \in\{1, \ldots, n-1\} \quad h_{*}^{i}\left(\alpha_{*}\right)=h^{i}(\alpha)$,

- $h_{*}^{n}\left(\alpha_{*}\right)=\left[h^{n}(p), x\right]_{h^{n}(\alpha)}$.

Proof. If $n=2$ then $\{x\}=\{p\}=\left\{h^{3}(p)\right\}=\alpha \cap h^{2}(\alpha)$ and there is nothing to prove. From now on we suppose $n \geq 3$.

STEP 1. If we already have $x=p$, just define $g=h$. Otherwise, since $x \neq h(p)$ by the minimality of $n$, observe that the $\operatorname{arc}[p, x]_{\alpha}$ has the following two properties:

(i) it is disjoint from its images under $h$ and $h^{2}$,

(ii) it is disjoint from $h^{i}(\alpha)$ for every $i \in\{1, \ldots, n-1\}$.

One can construct a topological closed disc $D_{1}$ which is a neighbourhood of $[p, x]_{\alpha}$ thin enough to satisfy (i) and (ii). Since $\alpha_{*}=[x, h(p)]_{\alpha}$ is an arc, one can also construct a homeomorphism $\varphi$ of $\mathbb{S}^{2}$ with support in $D_{1}$ such that $\varphi\left(\alpha_{*}\right)=\alpha$ (see for example [3, Lemma 2]). Defining $g=h \circ \varphi$, let us check that $g \sim h$ and also that $\alpha_{*}$ is a translation arc for $g$ with $g(x)=h(p)$ and

$$
\forall i \in\{1, \ldots, n\} \quad g^{i}\left(\alpha_{*}\right)=h^{i}(\alpha) .
$$

The Alexander trick gives an isotopy $\left(\varphi_{t}\right)_{0 \leq t \leq 1}$ with support in $D_{1}$ from $\varphi_{0}=\operatorname{Id}_{\mathbb{S}^{2}}$ to $\varphi_{1}=\varphi$. It is easily seen from $D_{1} \cap h\left(D_{1}\right)=\emptyset=D_{1} \cap h^{2}\left(D_{1}\right)$ that, for $i \in\{1,2\}$ and $t \in[0,1]$, the homeomorphisms $h^{i}$ and $\left(h \circ \varphi_{t}\right)^{i}$ have 
the same fixed point set. We also observe that

$$
z=h^{2}(z) \Rightarrow z \notin D_{1} \Rightarrow h \circ \varphi_{t}(z)=h(z) .
$$

Furthermore, if $\Omega \subset \mathbb{S}^{2}$ is an open set such that $\Omega \cap \operatorname{Fix}\left(h^{i}\right)$ is compact, we find that $\operatorname{Ind}\left(h^{i}, \Omega\right)=\operatorname{Ind}\left(g^{i}, \Omega\right)$ by considering the homotopy $\left(\left.\left(h \circ \varphi_{t}\right)^{i}\right|_{\Omega}\right)_{0 \leq t \leq 1}$ in Property 2.3(4). This shows $g \sim h$. By the construction we have $g\left(\alpha_{*}\right)=h \circ \varphi\left(\alpha_{*}\right)=h(\alpha)$ with $g(x)=h \circ \varphi(x)=h(p)$. Since $D_{1}$ is disjoint from $\bigcup_{i=1}^{n-1} h^{i}(\alpha)$, we get $g=h$ on $\bigcup_{i=1}^{n-1} h^{i}(\alpha)$ so

$$
\forall i \in\{1, \ldots, n\} \quad g^{i}\left(\alpha_{*}\right)=h^{i}(\alpha) \text { with } g^{i}(x)=h^{i}(p) .
$$

STEP 2. If $\{x\}=\left\{g^{n+1}(x)\right\}=\alpha_{*} \cap g^{n}\left(\alpha_{*}\right)$, it suffices to define $h_{*}=g$. Otherwise, since $x \neq g^{n}(x)=h^{n}(p)$, the arc $\left[x, g^{n+1}(x)\right]_{g^{n}\left(\alpha_{*}\right)}$ has the following properties:

(iii) it is disjoint from its images under $g$ and $g^{2}$,

(iv) it is disjoint from $g^{i}\left(\alpha_{*}\right)$ for every $i \in\{1, \ldots, n-1\}$.

Choose now a topological closed disc $D_{2}$ which is a neighbourhood of $\left[x, g^{n+1}(x)\right]_{g^{n}\left(\alpha_{*}\right)}$ satisfying (iii), (iv), and a homeomorphism $\psi$ of $\mathbb{S}^{2}$ supported in $D_{2}$ such that

$$
\psi\left(g^{n}\left(\alpha_{*}\right)\right)=\left[g^{n}(x), x\right]_{g^{n}\left(\alpha_{*}\right)} .
$$

Using the same arguments as in the first step, it is not difficult to check that $h_{*}=\psi \circ g \sim g$ has the required properties.

Lemma 3.4. Let $h, \alpha$ and $n$ be as in Lemma 3.3. Assume furthermore that $\alpha \cap h^{n}(\alpha)=\{p\}=\left\{h^{n+1}(p)\right\}$ and consider the Jordan curve $C=$ $\bigcup_{i=0}^{n} h^{i}(\alpha)$. If $U$ is a connected component of $\mathbb{S}^{2} \backslash C$, then $\operatorname{Ind}(h, U)=0$ and $\operatorname{Ind}\left(h^{2}, U\right)=1$.

Proof. It is easy to construct an orientation reversing homeomorphism $g$ of $\mathbb{S}^{2}$ with the following properties:

1. $g=h$ on $\bigcup_{i=0}^{n-1} h^{i}(\alpha)$,

2. $g$ maps $h^{n}(\alpha)$ onto $\alpha$ (hence $g(C)=C$ ),

3. $g$ interchanges the two connected components of $\mathbb{S}^{2} \backslash C$.

Thus $g^{-1} \circ h$ is an orientation preserving homeomorphism of the sphere which coincides with $\operatorname{Id}_{\mathbb{S}^{2}}$ on the arc $\bigcup_{i=0}^{n-1} h^{i}(\alpha)$. Using a variation of the Alexander trick (see for example [3, Lemma 1]) one can find an isotopy $\left(\varphi_{t}\right)_{0 \leq t \leq 1}$ from $\varphi_{0}=\operatorname{Id}_{\mathbb{S}^{2}}$ to $\varphi_{1}=g^{-1} \circ h$ such that

$$
\forall t \in[0,1] \forall z \in \bigcup_{i=0}^{n-1} h^{i}(\alpha) \quad \varphi_{t}(z)=z .
$$

Defining $h_{t}=g \circ \varphi_{t}(0 \leq t \leq 1)$, we obtain an isotopy from $h_{0}=g$ to $h_{1}=h$ such that $h_{t}=h$ on $\bigcup_{i=0}^{n-1} h^{i}(\alpha)$ and $\left(h_{t}^{2}\right)_{0 \leq t \leq 1}$ is then an isotopy 
from $g^{2}$ to $h^{2}$ such that $h_{t}^{2}=h^{2}$ on $\bigcup_{i=0}^{n-2} h^{i}(\alpha)$. Clearly $h^{2}$ has no fixed point on $\alpha$ and hence none on $\bigcup_{i \in \mathbb{Z}} h^{i}(\alpha)$. Consequently, for every $t \in[0,1]$, the homeomorphism $h_{t}^{2}$ (and so $h_{t}$ ) has no fixed point on

$$
\bigcup_{i=0}^{n-2} h^{i}(\alpha) \cup h_{t}\left(\bigcup_{i=0}^{n-2} h^{i}(\alpha)\right) \cup h_{t}^{2}\left(\bigcup_{i=0}^{n-2} h^{i}(\alpha)\right)=\bigcup_{i=0}^{n} h^{i}(\alpha)=C .
$$

Hence all the indices $\operatorname{Ind}\left(h_{t}, U\right)$ and $\operatorname{Ind}\left(h_{t}^{2}, U\right)$ are defined, and according to Property 2.3(4), we have $\operatorname{Ind}(g, U)=\operatorname{Ind}(h, U)$ and $\operatorname{Ind}\left(g^{2}, U\right)=\operatorname{Ind}\left(h^{2}, U\right)$. We conclude by observing that $U \cap g(U)=\emptyset$ gives $\operatorname{Ind}(g, U)=0$, and as is well known, $U=g^{2}(U)$ implies $\operatorname{Ind}\left(g^{2}, U\right)=1$.

REMARK 3.5. If $n \geq 3$ in Lemma 3.4, then $\alpha \cup h(\alpha)$ is a translation arc for the orientation preserving homeomorphism $h^{2}$, and Brouwer's lemma gives directly $\operatorname{Ind}\left(h^{2}, U\right)=1$.

Lemma 3.6. Let $h, \alpha, n$ and $C$ be as in Lemma 3.4. Then there exists a connected component $U$ of $\mathbb{S}^{2} \backslash C$ such that $\operatorname{Ind}\left(h^{2}, U \cap h(U)\right)=0$.

Proof. Let $U_{1}$ and $U_{2}=\mathbb{S}^{2} \backslash \mathrm{Cl}\left(U_{1}\right)$ be the two connected components of $\mathbb{S}^{2} \backslash C$. We can assume $U_{i} \cap h\left(U_{i}\right) \neq \emptyset$ for both $i=1$ and $i=2$ since otherwise the result is obvious. Let for example $U=U_{1}$. According to Lemma 2.4, it suffices to prove that $\operatorname{Ind}\left(h^{2}, V\right)=0$ for any given $V \in \pi_{0}(U \cap h(U))$. Since $h$ reverses the orientation, every point $z \in C \backslash h^{n}(\alpha)$ admits a neighbourhood $N_{z}$ such that $h\left(N_{z} \cap U\right)=h\left(N_{z}\right) \cap U_{2}$ and $h\left(N_{z} \cap U_{2}\right)=h\left(N_{z}\right) \cap U$. Consequently, $(C \backslash \alpha) \cap \mathrm{Cl}(U \cap h(U))=\emptyset$. In particular, $h^{ \pm 1}(U) \not \subset U$ and we obtain the following properties for every $V \in \pi_{0}(U \cap h(U))$ :

(1) $V \subset U$ with $V \neq U$,

(2) $V$ is a Jordan domain such that $\partial V \subset \alpha \cup h^{n+1}(\alpha)$,

(3) $\partial V \cap C$ contains at least two points.

The first one is clear since $U \not \subset h(U)$. We know from Proposition 2.1 that $V$ is a Jordan domain such that $\partial V \subset C \cup h(C)=\bigcup_{i=0}^{n+1} h^{i}(\alpha)$, and since $\mathrm{Cl}(V) \subset \mathrm{Cl}(U \cap h(U))$ is disjoint from $C \backslash \alpha$, we obtain more precisely $\partial V \subset \alpha \cup h^{n+1}(\alpha)$. The third property follows since otherwise we would have

$$
\partial V=\mathrm{Cl}(\partial V \backslash C) \subset h^{n+1}(\alpha),
$$

which is absurd because an arc cannot contain a Jordan curve.

Thus we can use Lemma 2.2. Every connected component $\mu$ of $U \cap \partial V$ is an open arc and property (2) above also shows that such a $\mu$ is a subset of $h^{n+1}(\alpha)$ and has endpoints in $\alpha$. Consequently, $C \backslash \alpha$ is contained in the frontier of one of the two connected components of $U \backslash \mu$ and is disjoint from the frontier of the other one (see Lemma 2.2(1)). Now, since $C \backslash \alpha \subset$ $C \backslash \mathrm{Cl}(V)$, there exists a path $\gamma$ from a point $a \in U$ to a point $b \in C \backslash \alpha$ such 
that $\gamma \backslash\{b\} \subset U \backslash \mathrm{Cl}(V)$. Let $\mu=\mu(a) \in \pi_{0}(U \cap \partial V)$ be such that $a \notin U_{\mu, V}$ (Lemma 2.2(2)). Thus $b$ is in the frontier of the connected component of $U \backslash \mu$ which does not contain $V$ so $(C \backslash \alpha) \cap \partial U_{\mu, V}=\emptyset$ and then $\mu_{*} \subset \alpha$.

We finally obtain $\operatorname{Ind}\left(h^{2}, V\right)=0$ by applying Lemma 2.6 with $f=h^{2}$ because

$$
\begin{gathered}
U \cap \partial V \cap h^{2}(U) \subset h^{n+1}(\alpha) \cap h^{2}(U)=h^{2}\left(h^{n-1}(\alpha) \cap U\right)=\emptyset, \\
h^{2}\left(\mu_{*}\right) \cap U \subset h^{2}(\alpha) \cap U=\emptyset .
\end{gathered}
$$

Proof of Proposition 3.2. We consider the integer $n \geq 2$ and the point $x \in h^{n}(\alpha)$ defined in Lemma 3.3. The set

$$
C=[x, h(p)]_{\alpha} \cup \bigcup_{i=1}^{n-1} h^{i}(\alpha) \cup\left[h^{n}(p), x\right]_{h^{n}(\alpha)}
$$

is then a Jordan curve. If necessary we can replace $h, \alpha$ with $h_{*}, \alpha_{*}$ given by Lemma 3.3 so there is no loss in supposing $x=p=h^{n+1}(p)$ and $C=$ $\bigcup_{i=0}^{n} h^{i}(\alpha)$. We complete the proof using Lemmas 3.4 and 3.6.

\subsection{A proposition about translation arcs of $h^{2}$}

Proposition 3.7. Let $h$ be an orientation reversing homeomorphism of $\mathbb{S}^{2}$. Assume that we can find a translation arc $\beta$ for $h^{2}$, with endpoints $q, h^{2}(q)$, such that

- $\beta \cap h(\beta)=\emptyset$,

- either $q=h^{4}(q)$ or $h^{k}(\beta) \cap \beta \neq \emptyset$ for an integer $k \geq 3$, i.e. the sets $\bigcup_{i \in \mathbb{Z}} h^{2 i}(\beta)$ and $\bigcup_{j \in \mathbb{Z}} h^{2 j+1}(\beta)$ are not two disjoint simple curves.

Then there exist a Jordan curve $C$ and a point $z$ as announced in Theorem 3.1 .

Beginning of the proof of Proposition 3.7. It will be convenient to define an integer $n \geq 2$ and a point $x \in h^{n}(\beta)$ as follows:

- if $q=h^{4}(q)$ then $n=2$ and $x=q=h^{4}(q)$,

- if $q \neq h^{4}(q)$ then $n$ is the minimum of the set $\left\{k \geq 3 \mid \beta \cap h^{k}(\beta) \neq \emptyset\right\}$ and $x$ is the first point on $h^{n}(\beta)$ to fall into $\beta$.

Let us remark that because of the minimality of $n$, we necessarily have $x \notin\left\{h^{2}(q), h^{n}(q)\right\}$. We also note that $h^{2}$ (and so $h$ ) has no fixed point on $\bigcup_{k \in \mathbb{Z}} h^{k}(\beta)$. The proof of Proposition 3.7 depends on the parity of $n$, as explained below.

3.2.1. $n$ is even. We consider the set

$$
C=\left[x, h^{2}(q)\right]_{\beta} \cup \bigcup_{2 i=2}^{n-2} h^{2 i}(\beta) \cup\left[h^{n}(q), x\right]_{h^{n}(\beta)} .
$$


It is a Jordan curve contained in $\bigcup_{2 i=0}^{n} h^{2 i}(\beta)$ (we simply have $C=\beta \cup h^{2}(\beta)$ if $n=2$ ). It follows from the minimality of $n$ that

$$
\left(\bigcup_{2 i=0}^{n} h^{2 i}(\beta)\right) \cap\left(\bigcup_{2 j+1=1}^{n-1} h^{2 j+1}(\beta)\right)=\emptyset .
$$

Hence $\bigcup_{2 j+1=1}^{n-1} h^{2 j+1}(\beta)$ is disjoint from $C$ and, by connectedness, it is contained in one of the two connected components $U_{1}, U_{2}$ of $\mathbb{S}^{2} \backslash C$, say in $U_{2}$. Thus we also have $\bigcup_{2 i=2}^{n} h^{2 i}(\beta) \subset h\left(U_{2}\right)$. Observe that this implies $h^{ \pm 1}\left(U_{1}\right) \not \subset U_{1}$ and $U_{2} \cap h\left(U_{2}\right) \neq \emptyset$. Since $\beta$ is a translation arc for $h^{2}$, Brouwer's lemma gives $\operatorname{Ind}\left(h^{2}, U_{1}\right)=1$ and $U_{1} \cap \operatorname{Fix}\left(h^{2}\right) \neq \emptyset$. We can suppose $U_{1} \cap h\left(U_{1}\right) \neq \emptyset$ since otherwise $\operatorname{Fix}(h) \cap U_{1}=\emptyset$, hence $\operatorname{Ind}\left(h, U_{1}\right)=0$, and every fixed point $z$ of $h^{2}$ in $U_{1}$ satisfies $h(z) \in U_{2}$. Writing simply $U=U_{1}$, we know from Proposition 2.1 that every connected component $V$ of $U \cap h(U)$ is a Jordan domain such that $\partial V \subset C \cup h(C)$. Since $\mathrm{Cl}(V) \subset \mathrm{Cl}(U) \cap \mathrm{Cl}(h(U))$ is disjoint from $\bigcup_{k=1}^{n} h^{k}(\beta)$ we get in fact

$$
\partial V \subset\left[x, h^{2}(q)\right]_{\beta} \cup\left[h^{n+1}(q), h(x)\right]_{h^{n+1}(\beta)} .
$$

Choosing a point $a \in U$ close enough to $C \cap \bigcup_{2 i=2}^{n} h^{2 i}(\beta)$ and considering $\mu=\mu(a) \in \pi_{0}(U \cap \partial V)$ such that $a \notin U_{\mu, V}$ (Lemma 2.2) one can check that necessarily $\mu_{*} \subset\left[x, h^{2}(q)\right]_{\beta}$ (see Fig. 3 ). All this can be done as in Lemma 3.6 and the details are left to the reader. Furthermore, since

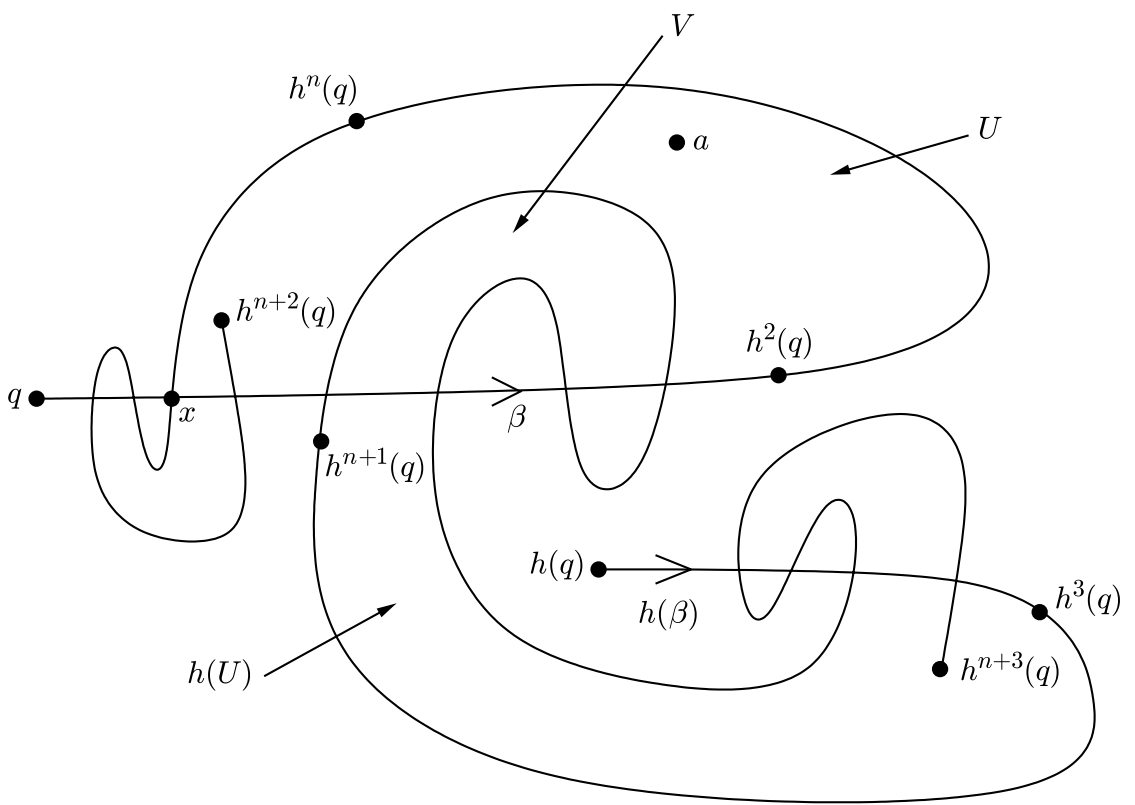

Fig. 3. The Jordan domains $U$ and $h(U)$ 


$$
\begin{aligned}
& U \cap \partial V \cap h(U) \subset \partial(U \cap h(U)) \cap U \cap h(U)=\emptyset, \\
& h\left(\mu_{*}\right) \cap U \subset h(\beta) \cap U=\emptyset,
\end{aligned}
$$

and

$$
\begin{aligned}
& U \cap \partial V \cap h^{2}(U) \subset h^{n+1}(\beta) \cap h^{2}(U)=h^{2}\left(h^{n-1}(\beta) \cap U\right)=\emptyset, \\
& h^{2}\left(\mu_{*}\right) \cap U \subset h^{2}(\beta) \cap U=\emptyset,
\end{aligned}
$$

one can use Lemma 2.6 with successively $f=h, f=h^{2}$ and thus obtain $\operatorname{Ind}(h, V)=0=\operatorname{Ind}\left(h^{2}, V\right)$. Since $\operatorname{Fix}(h) \cap U \cap h(U)=\operatorname{Fix}(h) \cap U$, by Property 2.3(1) and Lemma 2.4 we have

$$
\begin{aligned}
& 0=\sum_{V \in \pi_{0}(U \cap h(U))} \operatorname{Ind}(h, V)=\operatorname{Ind}(h, U \cap h(U))=\operatorname{Ind}(h, U), \\
& 0=\sum_{V \in \pi_{0}(U \cap h(U))} \operatorname{Ind}\left(h^{2}, V\right)=\operatorname{Ind}\left(h^{2}, U \cap h(U)\right) .
\end{aligned}
$$

This proves Proposition 3.7 when $n$ is even.

3.2.2. $n$ is odd and $h^{n+1}(\beta) \cap \beta=\emptyset$. We begin with a lemma which plays the same role as Lemma 3.3. Note that the assumption $h^{n+1}(\beta) \cap \beta=\emptyset$ is useless in this proof.

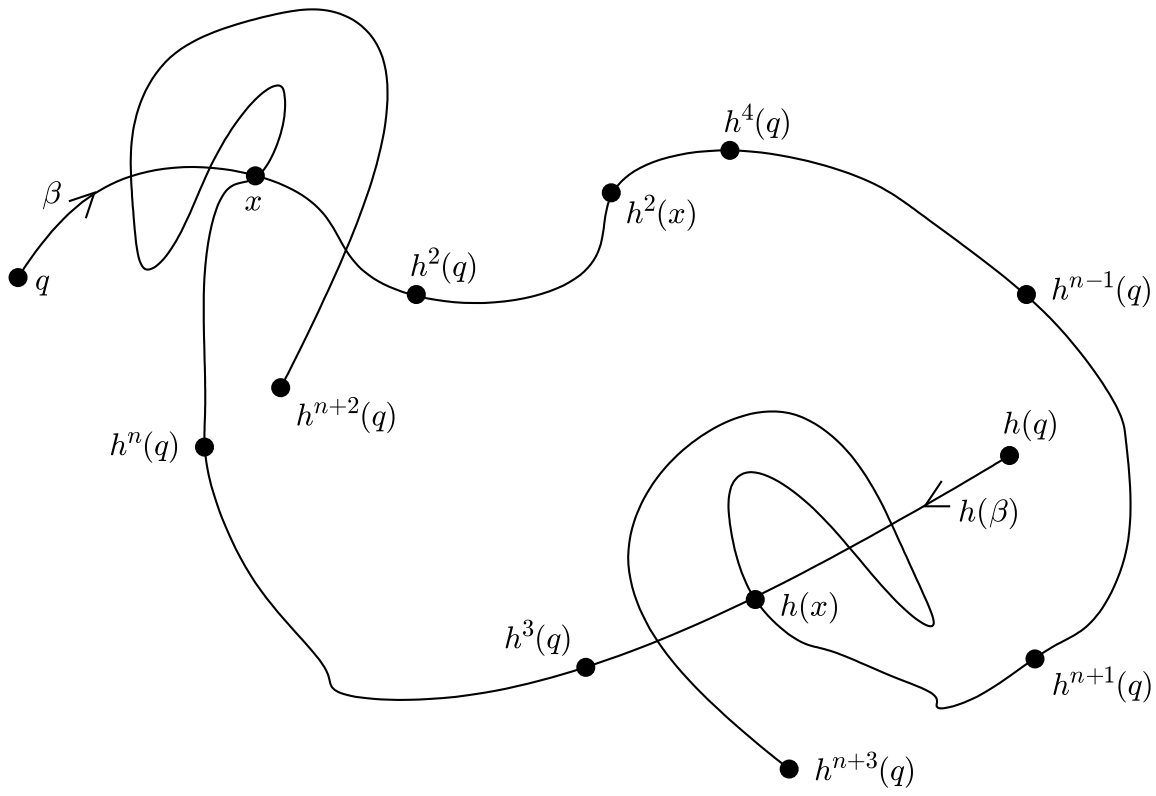

Fig. 4. The $\operatorname{arcs} h^{i}(\beta), 0 \leq i \leq n+1$ 
Lemma 3.8 (see Fig. 4). There exists an orientation reversing homeomorphism $h_{*} \sim h$ such that $h_{*}^{2}$ admits $\beta_{*}=\left[x, h^{2}(q)\right]_{\beta}$ as a translation arc with $h_{*}^{2}(x)=h^{2}(q)$ and

- $h_{*}\left(\beta_{*}\right)=\left[h(x), h^{3}(q)\right]_{h(\beta)}$,

- $\forall i \in\{2, \ldots, n-1\} \quad h_{*}^{i}\left(\beta_{*}\right)=h^{i}(\beta)$,

- $h_{*}^{n}\left(\beta_{*}\right)=\left[h^{n}(q), x\right]_{h^{n}(\beta)}$,

- $h_{*}^{n+1}\left(\beta_{*}\right)=\left[h^{n+1}(q), h(x)\right]_{h^{n+1}(\beta)}$.

Proof (outline). As in Lemma 3.3 the proof is in two steps.

STEP 1. If $x=q$ we rename $h=g$. Otherwise the $\operatorname{arc}\left[h^{2}(q), h^{2}(x)\right]_{h^{2}(\beta)}$ has the following properties:

(i) it is disjoint from its images under $h$ and $h^{2}$,

(ii) it is disjoint from $h^{i}(\beta)$ for every integer $i \in\{1\} \cup\{3, \ldots, n+1\}$.

One can construct a homeomorphism $\varphi$ of $\mathbb{S}^{2}$ mapping $\left[h^{2}(x), h^{4}(q)\right]_{h^{2}(\beta)}$ onto $h^{2}(\beta)$ whose support is contained in a topological closed disc $D_{1}$ so close to $\left[h^{2}(q), h^{2}(x)\right]_{h^{2}(\beta)}$ that it also satisfies (i) and (ii). Defining $g=\varphi \circ h$, we then have $g \sim h$ and $g=h$ on $\beta \cup \bigcup_{i=2}^{n} h^{i}(\beta)$, hence $g\left(\beta_{*}\right)=\left[h(x), h^{3}(q)\right]_{h(\beta)}$ with $g(x)=h(x)$ and

$$
\forall i \in\{2, \ldots, n+1\} \quad g^{i}\left(\beta_{*}\right)=h^{i}(\beta) \text { with } g^{i}(x)=h^{i}(p) .
$$

STEP 2. If $\{x\}=\left\{g^{n+2}(x)\right\}=\beta_{*} \cap g^{n}\left(\beta_{*}\right)$ it is enough to define $h_{*}=g$. Otherwise the arc $\left[x, g^{n+2}(x)\right]_{g^{n}\left(\beta_{*}\right)}$ is disjoint from its images under $g$ and $g^{2}$ and also from $\left(\bigcup_{i=1}^{n-1} g^{i}\left(\beta_{*}\right)\right) \cup g^{n+1}\left(\beta_{*}\right)$. It is possible to have the same for a topological closed disc $D_{2}$ containing the support of a homeomorphism $\psi$ of $\mathbb{S}^{2}$ such that $\psi\left(g^{n}\left(\beta_{*}\right)\right)=\left[g^{n}(x), x\right]_{g^{n}\left(\beta_{*}\right)}$. Then $h_{*}=\psi \circ g \sim g$ has the announced properties.

Continuation of the proof of Proposition 3.7. We now consider the sets

$$
\begin{aligned}
& \gamma_{-}=\left[x, h^{2}(q)\right]_{\beta} \cup \bigcup_{2 i=2}^{n-1} h^{2 i}(\beta) \cup\left[h^{n+1}(q), h(x)\right]_{h^{n+1}(\beta)}, \\
& \gamma_{+}=\left[h(x), h^{3}(q)\right]_{h(\beta)} \cup \bigcup_{2 j+1=3}^{n-2} h^{2 j+1}(\beta) \cup\left[h^{n}(q), x\right]_{h^{n}(\beta)}
\end{aligned}
$$

and finally $C=\gamma_{-} \cup \gamma_{+}$. Keeping in mind that $\beta \cap h^{n+1}(\beta)=\emptyset$, we see that $\gamma_{-}$and $\gamma_{+}$are two arcs which meet only in their common endpoints $x, h(x)$. Consequently, $C$ is a Jordan curve. Replacing $h, \beta$ with respectively $h_{*}, \beta_{*}$ given by Lemma 3.8 , one can suppose that $x=q=h^{n+2}(q)$, that is,

$$
\gamma_{-}=\bigcup_{2 i=0}^{n+1} h^{2 i}(\beta), \quad \gamma_{+}=\bigcup_{2 j+1=1}^{n} h^{2 j+1}(\beta), \quad C=\bigcup_{i=0}^{n+1} h^{i}(C) .
$$


Lemma 3.9. Let $U$ be a connected component of $\mathbb{S}^{2} \backslash C$. Then $\operatorname{Ind}(h, U)$ $=0$ and $\operatorname{Ind}\left(h^{2}, U\right)=1$.

Proof. It is similar to the one of Lemma 3.4. One can easily construct an orientation reversing homeomorphism $g$ of $\mathbb{S}^{2}$ such that

1. $g=h$ on the set $\bigcup_{i=0}^{n} h^{i}(\beta)$,

2. $g$ maps $h^{n+1}(\beta)$ onto $\beta$ (hence $g(C)=C$ ),

3. $g$ interchanges the two connected components of $\mathbb{S}^{2} \backslash C$.

Thus $g^{-1} \circ h$ is an orientation preserving homeomorphism of the sphere which coincides with $\operatorname{Id}_{\mathbb{S}^{2}}$ on the $\operatorname{arc} \bigcup_{i=0}^{n} h^{i}(\beta)$. Using the same variation of the Alexander trick as in Lemma 3.4, one can find an isotopy $\left(\varphi_{t}\right)_{0 \leq t \leq 1}$ from $\varphi_{0}=\operatorname{Id}_{\mathbb{S}^{2}}$ to $\varphi_{1}=g^{-1} \circ h$ such that

$$
\forall t \in[0,1] \forall z \in \bigcup_{i=0}^{n} h^{i}(\beta) \quad \varphi_{t}(z)=z .
$$

Defining $h_{t}=g \circ \varphi_{t}(0 \leq t \leq 1)$, we get an isotopy from $g$ to $h$ such that $h_{t}=h$ on $\bigcup_{i=0}^{n} h^{i}(\beta)$, and also an isotopy $\left(h_{t}^{2}\right)_{0 \leq t \leq 1}$ from $g^{2}$ to $h^{2}$ such that $h_{t}^{2}=h^{2}$ on $\bigcup_{i=0}^{n-1} h^{i}(\beta)$. It follows, for every $t \in[0,1]$, that $h_{t}^{2}$ has no fixed point on

$$
\bigcup_{i=0}^{n-1} h^{i}(\beta) \cup h_{t}^{2}\left(\bigcup_{i=0}^{n-1} h^{i}(\beta)\right)=\bigcup_{i=0}^{n+1} h^{i}(\beta)=C .
$$

Using again Property 2.3(4), we get $\operatorname{Ind}(g, U)=\operatorname{Ind}(h, U)$ and $\operatorname{Ind}\left(g^{2}, U\right)=$ $\operatorname{Ind}\left(h^{2}, U\right)$. We finally obtain $\operatorname{Ind}(g, U)=0\left(\right.$ resp. $\left.\operatorname{Ind}\left(g^{2}, U\right)=1\right)$ because $U \cap g(U)=\emptyset\left(\right.$ resp. $\left.U=g^{2}(U)\right)$.

Continuation of the proof of Proposition 3.7. Let $U_{1}, U_{2}$ be the two connected components of $\mathbb{S}^{2} \backslash C$. According to Lemma 3.9 we have $\operatorname{Ind}\left(h, U_{i}\right)=0$ and $\operatorname{Ind}\left(h^{2}, U_{i}\right)=1$. In particular $U_{i} \cap \operatorname{Fix}\left(h^{2}\right) \neq \emptyset(i \in\{1,2\})$. If one can find $i \in\{1,2\}$ such that $U_{i} \cap h\left(U_{i}\right)=\emptyset$ then the result is easy. Otherwise we consider for example $U=U_{1}$. Let $V$ be any connected component of $U \cap h(U)$. Since $h$ reverses the orientation, every point $z \in C \backslash h^{n+1}(\beta)$ has a neighbourhood $N_{z}$ such that $h\left(N_{z} \cap U\right)=h\left(N_{z}\right) \cap U_{2}$ and $h\left(N_{z} \cap U_{2}\right)=h\left(N_{z}\right) \cap U$. It follows that $C \backslash \beta$ is disjoint from $\mathrm{Cl}(U \cap h(U))$ and in particular from $\mathrm{Cl}(V)$. Using Proposition 2.1 one more time, we deduce that $V$ is a Jordan domain such that $\partial V \subset \beta \cup h^{n+2}(\beta)$. As in the proof of Lemma 3.6 one can use Lemma 2.2 to find $\mu \in \pi_{0}(U \cap \partial V)$ such that the corresponding arc $\mu_{*}$ satisfies $\mu_{*} \subset \beta$. We then have

$$
\begin{aligned}
& \partial V \cap U \cap h^{2}(U) \subset h^{n+2}(\beta) \cap h^{2}(U)=h^{2}\left(h^{n}(\beta) \cap U\right)=\emptyset, \\
& h^{2}\left(\mu_{*}\right) \cap U \subset h^{2}(\beta) \cap U=\emptyset,
\end{aligned}
$$


and Lemma 2.6 gives $\operatorname{Ind}\left(h^{2}, V\right)=0$. We deduce from Lemma 2.4 that

$$
0=\sum_{V \in \pi_{0}(U \cap h(U))} \operatorname{Ind}\left(h^{2}, V\right)=\operatorname{Ind}\left(h^{2}, U \cap h(U)\right) .
$$

3.2.3. $n$ is odd and $h^{n+1}(\beta) \cap \beta \neq \emptyset$. The following remarks allow us to reduce the proof to the cases studied in Sections 3.2.1 and 3.2.2. We consider the last point $y$ on $\beta$ to fall into $h^{n}(\beta) \cup h^{n+1}(\beta)$. Since $\beta \cap h(\beta)=\emptyset, y$ does not belong to $h^{n}(\beta)$ and $h^{n+1}(\beta)$ simultaneously, and $y \neq q$. We also have $y \neq h^{2}(q)$ because of the minimality of $n$. We can then assert:

LEMMA 3.10. There exists an orientation reversing homeomorphism $\widehat{h} \sim$ $h$ such that $\widehat{h}^{2}$ admits $\widehat{\beta}=\left[y, h^{2}(q)\right]_{\beta}$ as a translation arc with $\widehat{h}^{2}(y)=h^{2}(q)$ and

- $\widehat{h}(\widehat{\beta})=\left[h(y), h^{3}(q)\right]_{h(\beta)}$,

- $\forall i \in\{2, \ldots, n+1\} \widehat{h}^{i}(\widehat{\beta})=h^{i}(\beta)$.

Proof. It is enough to replace $x$ with $y$ in the construction of the intermediate homeomorphism $g$ in the proof of Lemma 3.8.

End of the proof of Proposition 3.7. By the definition of $y$ we have:

- if $y \in h^{n}(\beta)=\widehat{h}^{n}(\widehat{\beta})$ then $\widehat{h}^{n+1}(\widehat{\beta}) \cap \widehat{\beta}=h^{n+1}(\beta) \cap \widehat{\beta}=\emptyset$ and we reduce to the situation of Section 3.2 .2 by replacing $h, \beta$ with $\widehat{h}, \widehat{\beta}$;

- if $y \in h^{n+1}(\beta)=\widehat{h}^{n+1}(\widehat{\beta})$ then $\widehat{h}^{4}(y)=h^{4}(q) \neq y$ and $n+1$ is the smallest integer $k \in\{3, \ldots, n+1\}$ such that $\widehat{h}^{k}(\widehat{\beta})=h^{k}(\beta)$ intersects $\widehat{\beta}$. We reduce to the case treated in Section 3.2.1 by replacing $h, \beta$ and $n$ with $\widehat{h}, \widehat{\beta}$ and $n+1$.

Proposition 3.7 is proved.

3.3. Proof of Theorem 3.1. Choose $m$ to be a $k$-periodic point of $h(k \geq 3)$ and consider an arc $\alpha$ or $\beta$ given by Lemma 2.8. Then $m=$ $h^{k}(m) \in \alpha \cap h^{k}(\alpha)$ or $m=h^{k}(m) \in \beta \cap h^{k}(\beta)$ and either Proposition 3.2 or Proposition 3.7 applies.

\subsection{Some consequences}

COROLLARY 3.11. Let $h$ be an orientation reversing homeomorphism of the sphere $\mathbb{S}^{2}$ without a 2-periodic point. If a connected and simply connected compact set $K \subset \mathbb{S}^{2}$ satisfies $K \cap h(K)=\emptyset=K \cap h^{2}(K)$ then $K \cap h^{k}(K)=\emptyset$ for every integer $k \neq 0$. Consequently, the only non-wandering points are the fixed points of $h$.

Proof. Take a topological closed disc $D$ containing $K$ and so close to $K$ that it is disjoint from its iterates $h(D)$ and $h^{2}(D)$. We claim that $D \cap$ $h^{k}(D)=\emptyset$ for every integer $k \neq 0$; otherwise, there exists a smallest $k \geq 3$ 
such that $D \cap h^{k}(D) \neq \emptyset$. Replacing the disc $D$ with a slightly larger one if necessary, we can suppose $\operatorname{Int}(D) \cap h^{k}(\operatorname{Int}(D)) \neq \emptyset$. Then we can choose $m \in \operatorname{Int}(D) \cap h^{-k}(\operatorname{Int}(D))$ and a homeomorphism $\varphi$ with support in $D$ such that $\varphi\left(h^{k}(m)\right)=m$. Thus $\varphi \circ h$ is an orientation reversing homeomorphism of $\mathbb{S}^{2}$ with $m$ as a $k$-periodic point. According to Theorem 3.1, $\varphi \circ h$ admits a 2-periodic point. The support of $\varphi$ is disjoint from its images under $h$ and $h^{2}$ so, for $i \in\{1,2\}$, the homeomorphisms $(\varphi \circ h)^{i}$ and $h^{i}$ have the same fixed point set. Thus we get a 2-periodic point for $h$, a contradiction.

Now, for any point $m \in \mathbb{S}^{2} \backslash \operatorname{Fix}(h)=\mathbb{S}^{2} \backslash \operatorname{Fix}\left(h^{2}\right)$ we can choose $K$ to be a neighbourhood of $m$ so $m$ is a wandering point.

REMARK 3.12. As an immediate consequence of Corollary 3.11, any area preserving and orientation reversing homeomorphism of the 2-sphere (or of the closed 2-disc) has a 2-periodic point.

REMARK 3.13. If in Theorem 3.1 we assume furthermore that $h^{2}$ has only finitely many fixed points then the 2-periodic point $z$ can be chosen such that $\operatorname{Ind}\left(h^{2}, z\right)=\operatorname{Ind}\left(h^{2}, h(z)\right)$ is a positive integer (resp. an odd integer).

The equality $\operatorname{Ind}\left(h^{2}, z\right)=\operatorname{Ind}\left(h^{2}, h(z)\right)$ is a consequence of Property 2.3(5) and of the obvious relation $h^{2}=h \circ h^{2} \circ h^{-1}$. Keeping the notations of Theorem 3.1 define

$F=\operatorname{Fix}\left(h^{2}\right) \cap U, \quad F_{1}=F \cap h(U), \quad F_{2}=F \backslash F_{1}=\operatorname{Fix}\left(h^{2}\right) \cap U \cap h^{-1}\left(U^{\prime}\right)$.

Then

$$
1=\operatorname{Ind}\left(h^{2}, U\right)=\sum_{z \in F} \operatorname{Ind}\left(h^{2}, z\right)=\sum_{z \in F_{1}} \operatorname{Ind}\left(h^{2}, z\right)+\sum_{z \in F_{2}} \operatorname{Ind}\left(h^{2}, z\right) .
$$

Recall we have shown $\operatorname{Ind}\left(h^{2}, U \cap h(U)\right)=0$, hence $\sum_{z \in F_{1}} \operatorname{Ind}\left(h^{2}, z\right)=0$ and the assertion follows.

\section{A LOCAL VERSION OF THEOREM 3.1}

We first recall two recent results:

TheOREM 4.1 ([1]). Let $V, W$ be two connected open subsets of $\mathbb{R}^{2}$ containing the origin o and let $h: V \rightarrow W=h(V)$ be an orientation reversing homeomorphism for which $o$ is an isolated fixed point. Then $\operatorname{Ind}(h, o) \in$ $\{-1,0,1\}$.

The iterated homeomorphisms $h^{k}: V_{k} \rightarrow h_{k}\left(V_{k}\right), k \geq 1$, are defined inductively on the open sets $V_{k}$ by $V_{1}=V, h^{1}=h$ and, for $k \geq 2, V_{k}=$ $h^{-1}\left(V_{k-1}\right) \subset V_{k-1}, h^{k}(z)=h^{k-1}(h(z))$.

We then have 
Theorem 4.2 ([11]). Let $h$ be as in Theorem 4.1. Assume that the whole sequence $\left(\operatorname{Ind}\left(h^{k}, o\right)\right)_{k \geq 1}$ is defined, i.e. o is an isolated fixed point of $h^{k}$ for every integer $k \geq 1$. Then $\left(\operatorname{Ind}\left(h^{2 k+1}, o\right)\right)_{k \geq 0}$ is a constant sequence.

We can now state:

THEOREM 4.3. Let $h$ be as in Theorem 4.1. If there exists an integer $k \geq 3$ such that any neighbourhood of o contains a $k$-periodic point of $h$ then there is also a 2-periodic point in every neighbourhood of o. In other words, the whole sequence $\left(\operatorname{Ind}\left(h^{k}, o\right)\right)_{k \geq 1}$ is defined if and only if the second term $\operatorname{Ind}\left(h^{2}, o\right)$ is defined.

Proof. Let $\Omega$ be an open disc with center $o$, so small that $\Omega \cap \operatorname{Fix}(h)=$ $\{o\}$ and $\mathrm{Cl}(\Omega) \subset V$. Let us show that $\Omega$ necessarily contains a 2-periodic point of $h$. Using the Schoenflies Theorem, we can extend $\left.h\right|_{\mathrm{Cl}(\Omega)}$ to a homeomorphism $H$ of the whole sphere. Let $\Omega^{\prime}$ be an open disc with center $o$ such that $\Omega^{\prime} \subset \Omega \cap H^{-1}(\Omega)$ and let $N$ be the connected component of $\bigcap_{i=0}^{k+1} H^{-i}\left(\Omega^{\prime}\right)$ containing $o$. The set $N \cap H^{-2}(N)$ is a neighbourhood of $o$ so it contains a $k$-periodic point $m$ of $h$, and such a point $m$ is also $k$-periodic for $H$ because $h^{j}=H^{j}$ on $\bigcap_{i=0}^{k-1} H^{-i}\left(\Omega^{\prime}\right)$ for $j=1, \ldots, k$. Since $h=H$ and $h^{2}=H^{2}$ on $\Omega \cap H^{-1}(\Omega)$ it is enough to prove that $H$ has a 2-periodic point in $\Omega^{\prime}$. Suppose this is not true. Then we have

$$
\left\{m, H^{2}(m)\right\} \subset N \backslash\{o\}=N \backslash \operatorname{Fix}\left(H^{2}\right) .
$$

The contradiction is obtained by following carefully the proof of Theorem 3.1 for the homeomorphism $H$. Since an open connected subset of $\mathbb{R}^{2}$ is arcwise connected, there exists an arc $\gamma \subset N \backslash\{o\}$ joining $m$ and $H^{2}(m)$. Going back to the proof of Lemma 2.8, we now slightly enlarge $\gamma$ to obtain a topological closed disc $\Delta$ such that $\gamma \subset \operatorname{Int}(\Delta) \subset \Delta \subset N \backslash\{o\}$. Then we obtain a suitable translation arc for either $H$ or $H^{2}$, which is denoted by respectively $\alpha$ or $\beta$. Observe that, by the construction, $\alpha$ and $\beta$ are contained in $\Delta$ and so in $\bigcap_{i=0}^{k+1} H^{-i}\left(\Omega^{\prime}\right)$. Note finally that all the Jordan curves $C$ constructed in the proof of Theorem 3.1 are subsets of $\bigcup_{i=0}^{k} H^{i}(\alpha) \subset \Omega^{\prime}$ or of $\bigcup_{i=0}^{k+1} H^{i}(\beta) \subset \Omega^{\prime}$, hence $\mathbb{S}^{2} \backslash C$ has a connected component which is contained in the disc $\Omega^{\prime}$. Thus we obtain a 2-periodic point of $H$ in $\Omega^{\prime}$, a contradiction.

\section{A STRONG VERSION OF THEOREM 3.1}

In this section we prove the following result:

THEOREM 5.1. Let $h$ be an orientation reversing homeomorphism of the sphere $\mathbb{S}^{2}$ without a 2-periodic point. Then for any point $m \in \mathbb{S}^{2} \backslash \operatorname{Fix}(h)$ there exists a topological embedding (i.e. a continuous one-to-one map) $\varphi$ : $\mathcal{O} \rightarrow \mathbb{S}^{2} \backslash \operatorname{Fix}(h)$ such that 
- $\mathcal{O}$ is either $\mathbb{R}^{2}$ or $\left\{(x, y) \in \mathbb{R}^{2} \mid y \neq 0\right\}$ or $\mathbb{R}^{2} \backslash\{(0,0)\}$,

- $m \in \varphi(\mathcal{O})$,

- if $\mathcal{O}=\mathbb{R}^{2}$ or $\mathcal{O}=\left\{(x, y) \in \mathbb{R}^{2} \mid y \neq 0\right\}$ then

(i) $h \circ \varphi=\left.\varphi \circ G\right|_{\mathcal{O}}$, where $G(x, y)=(x+1,-y)$,

(ii) for every $x \in \mathbb{R}, \varphi((\{x\} \times \mathbb{R}) \cap \mathcal{O})$ is a closed subset of $M \backslash \operatorname{Fix}(h)$ (we say that $\varphi$ is a proper embedding),

- if $\mathcal{O}=\mathbb{R}^{2} \backslash\{(0,0)\}$ then

(iii) $h \circ \varphi=\left.\varphi \circ H\right|_{\mathcal{O}}$, where $H(x, y)=\frac{1}{2}(x,-y)$.

Sections 5.2 and 5.3 below contain preparatory results. Section 5.2 gives some dynamical properties of orientation reversing homeomorphisms of the sphere, derived from the results of Section 3. In particular it is shown that recurrence of discs, just as recurrence of points, implies the existence of a 2-periodic point. Section 5.3 recalls the notion of brick decomposition of a surface introduced by P. Le Calvez and A. Sauzet ([16], [19]) to give a dynamical proof of the Brouwer plane translation theorem. Theorem 5.1 is proved in Section 5.3.

Note that since we are looking for conjugacy outside the fixed point set, the map $H$ in the statement of Theorem 5.1 can be replaced with any map $(x, y) \mapsto \lambda(x,-y)$, where $\lambda \in \mathbb{R} \backslash\{ \pm 1\}$.

\subsection{Some recurrence properties}

LEMMA 5.2. Let $h$ be an orientation reversing homeomorphism of $\mathbb{S}^{2}$ without a 2-periodic point and let $V$ be an open connected subset of $\mathbb{S}^{2}$ such that $V \cap h(V)=\emptyset=V \cap h^{2}(V)$. Then $V \cap h^{k}(V)=\emptyset$ for any integer $k \neq 0$.

Proof. Suppose this is not true. Then $V \cap h^{k}(V) \neq \emptyset$ for an integer $k \geq 3$, so choose $z \in V \cap h^{k}(V)$. Since an open connected subset of $\mathbb{S}^{2}$ is arcwise connected, there exists an arc $K \subset V$ with endpoints $h^{-k}(z)$ and $z$. Such an arc is disjoint from its first two iterates $h(K), h^{2}(K)$ but meets $h^{k}(K)$. This contradicts Corollary 3.11.

The next lemmas can be regarded as the counterpart of Frank's lemma ([8, Proposition 1.3]) in the case of an orientation reversing homeomorphism.

Lemma 5.3. Let $h$ be an orientation reversing homeomorphism of $\mathbb{S}^{2}$. Assume that there exists a finite sequence of topological closed discs $D_{1}, \ldots$ $\ldots, D_{n}$ satisfying

(i) $\forall i, j \in\{1, \ldots, n\} \quad D_{i}=D_{j}$ or $\operatorname{Int}\left(D_{i}\right) \cap \operatorname{Int}\left(D_{j}\right)=\emptyset$,

(ii) $\forall i \in\{1, \ldots, n\} \quad h\left(D_{i}\right) \cap D_{i}=\emptyset=h^{2}\left(D_{i}\right) \cap D_{i}$,

(iii) $\forall i, j \in\{1, \ldots, n\} \quad D_{j}$ meets at most one of the two sets $h^{-1}\left(D_{i}\right)$ or $h\left(D_{i}\right)$, equivalently: $h\left(D_{i}\right) \cap D_{j} \neq \emptyset \Rightarrow h\left(D_{j}\right) \cap D_{i}=\emptyset$, 
(iv) $\forall i \in\{1, \ldots, n-1\} \exists k_{i} \geq 1$ such that $h^{k_{i}}\left(D_{i}\right) \cap \operatorname{Int}\left(D_{i+1}\right) \neq \emptyset$ and $\exists k_{n} \geq 1$ such that $h^{k_{n}}\left(D_{n}\right) \cap \operatorname{Int}\left(D_{1}\right) \neq \emptyset$.

Then $h$ has a 2-periodic point.

Proof. Take a sequence $D_{1}, \ldots, D_{n_{0}}$ satisfying (i)-(iv) and whose length $n_{0}$ is minimal among all such sequences. If $n_{0}=1$ then the result is contained in Corollary 3.11 so we can assume $n_{0} \geq 2$. Moreover we can suppose that the integers $k_{1}, \ldots, k_{n_{0}}$ are minimal for property (iv). In order to simplify the notations we also define $D_{n_{0}+1}=D_{1}$. We have clearly

$$
h^{k_{i}}\left(D_{i}\right) \cap \operatorname{Int}\left(D_{i+1}\right) \neq \emptyset \Leftrightarrow h^{k_{i}}\left(\operatorname{Int}\left(D_{i}\right)\right) \cap \operatorname{Int}\left(D_{i+1}\right) \neq \emptyset
$$

so for every $i \in\left\{1, \ldots, n_{0}\right\}$ we can choose $x_{i} \in \operatorname{Int}\left(D_{i}\right)$ such that $h^{k_{i}}\left(x_{i}\right) \in$ $\operatorname{Int}\left(D_{i+1}\right)$. Since the sequence $D_{1}, \ldots, D_{n_{0}}$ has minimal length we have

$$
1 \leq i \neq j \leq n_{0} \Rightarrow \operatorname{Int}\left(D_{i}\right) \cap \operatorname{Int}\left(D_{j}\right)=\emptyset
$$

so there exists an orientation preserving homeomorphism $\psi$ of $\mathbb{S}^{2}$ with support in $D_{1} \cup \cdots \cup D_{n_{0}}$ preserving setwise each disc $D_{i}\left(1 \leq i \leq n_{0}\right)$ and such that

$$
\forall i \in\left\{1, \ldots, n_{0}-1\right\} \quad \psi\left(h^{k_{i}}\left(x_{i}\right)\right)=x_{i+1}, \quad \psi\left(h^{k_{n_{0}}}\left(x_{n_{0}}\right)\right)=x_{1} .
$$

Furthermore for every $i, j \in\left\{1, \ldots, n_{0}\right\}$ we have

$$
1 \leq k \leq k_{i}-1 \Rightarrow h^{k}\left(x_{i}\right) \notin D_{j}
$$

since otherwise either $D_{1}, \ldots, D_{i}, D_{j}, \ldots, D_{n_{0}}$ or $D_{j}, \ldots, D_{i}$ would define a sequence satisfying (i)-(iv) with length $\leq n_{0}-1$. Thus the homeomorphism $g=\psi \circ h$ reverses the orientation and has $x_{1}$ as a periodic point with period $k_{1}+\cdots+k_{n_{0}} \geq 2$. Theorem 3.1 then gives a 2-periodic point for $g$ and it is enough to check that $\operatorname{Fix}(h)=\operatorname{Fix}(g)$ and $\operatorname{Fix}\left(h^{2}\right)=\operatorname{Fix}\left(g^{2}\right)$.

The first equality is well known and follows easily from the fact that $D_{i} \cap h\left(D_{i}\right)=\emptyset$ for every $i \in\left\{1, \ldots, n_{0}\right\}$.

Let us check that $\operatorname{Fix}\left(g^{2}\right)=\operatorname{Fix}\left(h^{2}\right)$.

First we observe that if $m \in h^{-1}\left(D_{j}\right)$ for an index $j \in\left\{1, \ldots, n_{0}\right\}$ then necessarily $m \neq g^{2}(m)$ : for such an $m$ we have $g(m)=\psi(h(m)) \in \psi\left(D_{j}\right)$ $=D_{j}$ so $h(g(m)) \in h\left(D_{j}\right)$. If $h(g(m)) \notin \bigcup_{i=1}^{n_{0}} D_{i}$ then $g^{2}(m)=\psi(h(g(m)))=$ $h(g(m))$ and consequently $m \neq g^{2}(m)$ since $h^{-1}\left(D_{j}\right) \cap h\left(D_{j}\right)=\emptyset$. If one can find $i \in\left\{1, \ldots, n_{0}\right\}$ such that $h(g(m)) \in D_{i}$ then we obtain $h(g(m)) \in D_{i} \cap$ $h\left(D_{j}\right) \neq \emptyset$ and (iii) implies $D_{i} \cap h^{-1}\left(D_{j}\right)=\emptyset$. Since $g^{2}(m)=\psi(h(g(m))) \in$ $\psi\left(D_{i}\right)=D_{i}$, it follows that $g^{2}(m) \neq m$.

Secondly we remark that if $m \notin \bigcup_{i=1}^{n_{0}} h^{-1}\left(D_{i}\right)$ but $m \in h^{-2}\left(D_{j}\right)$ for a $j \in\left\{1, \ldots, n_{0}\right\}$ then we also have $m \neq g^{2}(m)$. Indeed, we then have $g(m)=$ $h(m), g^{2}(m)=\psi\left(h^{2}(m)\right) \in \psi\left(D_{j}\right)=D_{j}$ and consequently $m \neq g^{2}(m)$ since $h^{-2}\left(D_{j}\right) \cap D_{j}=\emptyset$. 
Thus we obtain

$$
m=g^{2}(m) \Rightarrow m \notin\left(\bigcup_{i=1}^{n_{0}} h^{-1}\left(D_{i}\right)\right) \cup\left(\bigcup_{i=1}^{n_{0}} h^{-2}\left(D_{i}\right)\right) \Rightarrow g^{2}(m)=h^{2}(m) \text {. }
$$

On the other hand, it is easily seen from (ii) that

$m=h^{2}(m) \Rightarrow m \notin\left(\bigcup_{i=1}^{n_{0}} h^{-1}\left(D_{i}\right)\right) \cup\left(\bigcup_{i=1}^{n_{0}} h^{-2}\left(D_{i}\right)\right) \Rightarrow g^{2}(m)=h^{2}(m)$.

We will actually use the following slightly stronger lemma which relaxes hypothesis (iv) of Lemma 5.3. This technical improvement allows us to suppress a hypothesis of "transversality" for the brick decompositions of a surface appearing in the original work of Le Calvez and Sauzet [16] (see Section 5.2 below for a definition). These refinements are due to F. Le Roux [17] for orientation preserving homeomorphisms. We use the same arguments to give a proof adapted to our situation.

LEMma 5.4. If in Lemma 5.3 we replace condition (iv) with the weaker $\left(\right.$ iv $\left.^{\prime}\right) \quad \forall i \in\{1, \ldots, n-1\} \exists k_{i} \geq 1 \quad h^{k_{i}}\left(D_{i}\right) \cap D_{i+1} \neq \emptyset$ and $\exists k_{n} \geq 1 h^{k_{n}}\left(D_{n}\right) \cap D_{1} \neq \emptyset$,

then the conclusion still holds.

Proof. Let $D_{1}, \ldots, D_{n_{0}}$ be a sequence of topological closed discs satisfying (i)-(iii), (iv') and whose length $n_{0}$ is minimal among all such sequences. Then

$$
i \neq j \Rightarrow \operatorname{Int}\left(D_{i}\right) \cap \operatorname{Int}\left(D_{j}\right)=\emptyset
$$

For each $i \in\left\{1, \ldots, n_{0}\right\}$ choose $x_{i} \in D_{i}$ such that $h^{k_{i}}\left(x_{i}\right) \in D_{i+1}$ (again with the convention $D_{n_{0}+1}=D_{1}$ ).

First we observe that the points $x_{1}, \ldots, x_{n_{0}}$ are distinct because

$$
\left(1 \leq i, j \leq n_{0} \text { and } x_{i}=x_{j}\right) \Rightarrow h^{k_{j}}\left(x_{i}\right)=h^{k_{j}}\left(x_{j}\right) \in h^{k_{j}}\left(D_{i}\right) \cap D_{j+1}
$$

and the fact that $D_{1}, \ldots, D_{n_{0}}$ has minimal length gives $i=j$.

Now, if we can find $i, j \in\left\{1, \ldots, n_{0}\right\}$ and an integer $k \geq 1$ such that $h^{k}\left(x_{i}\right)=x_{j}$ then $h$ has a 2 -periodic point. Indeed, then

$$
h^{k_{j}+k}\left(x_{i}\right)=h^{k_{j}}\left(x_{j}\right) \in h^{k_{j}+k}\left(D_{i}\right) \cap D_{j+1} .
$$

Again because $D_{1}, \ldots, D_{n_{0}}$ has minimal length, this is possible only for $i=j$ and consequently $h^{k}\left(x_{i}\right)=x_{i}$. Because of (ii) we necessarily have $k \geq 3$ and Theorem 3.1 then gives a 2-periodic point for $h$. Thus we can assume without loss of generality

$$
\forall i, j \in\left\{1, \ldots, n_{0}\right\} \forall k \neq 0 \quad h^{k}\left(x_{i}\right) \neq x_{j} .
$$

For convenience we let $k_{0}=k_{n_{0}}$ and $x_{0}=x_{n_{0}}$. Then for each $i \in\left\{1, \ldots, n_{0}\right\}$ we choose an arc $\gamma_{i}$ with endpoints $x_{i}$ and $h^{k_{i-1}}\left(x_{i-1}\right) \neq x_{i}$ lying entirely in 
$\operatorname{Int}\left(D_{i}\right)$ except possibly for its endpoints in $\partial D_{i}$. Since $\gamma_{i} \subset D_{i}$, these $\operatorname{arcs}$ have the same properties (ii), (iii) as the discs $D_{i}$. Moreover, remembering that the $D_{i}$ 's have disjoint interiors and the $x_{i}$ 's are distinct $\left(1 \leq i \leq n_{0}\right)$, we obtain, using $(* *)$,

(i') $i \neq j \Rightarrow \gamma_{i} \cap \gamma_{j}=\emptyset$.

By the construction we also have

$\forall i \in\left\{1, \ldots, n_{0}-1\right\} \quad h^{k_{i}}\left(\gamma_{i}\right) \cap \gamma_{i+1} \neq \emptyset \quad$ and $\quad h^{k_{n_{0}}}\left(\gamma_{n_{0}}\right) \cap \gamma_{1} \neq \emptyset$.

For each $i \in\left\{1, \ldots, n_{0}\right\}$ one can find a topological closed disc $D_{i}^{\prime}$ which is a neighbourhood of $\gamma_{i}$ so close to $\gamma_{i}$ that $\left(\mathrm{i}^{\prime}\right)$, (ii), (iii) are still true with the $D_{i}^{\prime}$ 's in place of the $\gamma_{i}^{\prime}$ 's. The sequence $D_{1}^{\prime}, \ldots, D_{n_{0}}^{\prime}$ then satisfies conditions (i)-(iv) of Lemma 5.3.

5.2. Brick decompositions. As mentioned above, this notion is due to P. Le Calvez and A. Sauzet ([16], [19]). It is also used with some variants in [13], [15] and [17].

Definition 5.5. A brick decomposition $\mathcal{D}$ of a nonempty open set $U \subset$ $\mathbb{S}^{2}$ is a collection $\left\{B_{i}\right\}_{i \in I}$ of topological closed discs, where $I$ is a finite or countable set, such that

1. $\bigcup_{i \in I} B_{i}=U$,

2. if $i \neq j$ then $B_{i} \cap B_{j}$ is either empty or an arc contained in $\partial B_{i} \cap \partial B_{j}$,

3. for every point $z \in U$, the set $I(z)=\left\{i \in I \mid z \in B_{i}\right\}$ contains at most three elements and $\bigcup_{i \in I(z)} B_{i}$ is a neighbourhood of $z$ in $U$.

The $B_{i}$ 's are called the bricks of the decomposition. Of course the set $I$ is finite only for $U=\mathbb{S}^{2}$ and we will not be concerned with this situation. For a point $z \in U$, the neighbourhood $\bigcup_{i \in I(z)} B_{i}$ is necessarily of one of the three kinds pictured in Fig. 5 (up to homeomorphism).
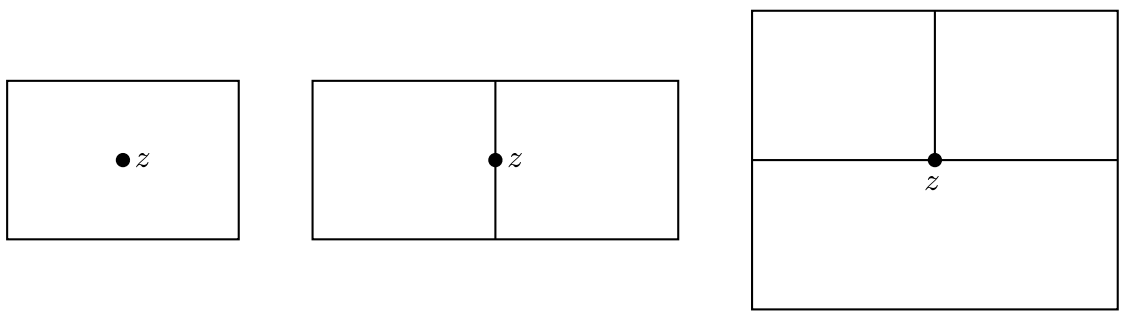

Fig. 5. The neighbourhood $\bigcup_{i \in I(z)} B_{i}$ for a point $z \in U$

We then have the following property which is one of the main motivations for the use of brick decompositions. 
Property 5.6. Let $\mathcal{D}=\left\{B_{i}\right\}_{i \in I}$ be a brick decomposition of an open set $U \subset \mathbb{S}^{2}$ and let $J$ be a nonempty subset of $I$. Then $\bigcup_{i \in J} B_{i}$ is a closed subset of $U$. Furthermore $\partial_{U}\left(\bigcup_{i \in J} B_{i}\right)$ is a 1-dimensional submanifold of $U$ without boundary. In particular, its connected components are homeomorphic either to $\mathbb{S}^{1}$ or to $\mathbb{R}$.

Proof. If $z \in \mathrm{Cl}_{U}\left(\bigcup_{i \in J} B_{i}\right)$ it is clear from the definition that $I(z) \cap J \neq \emptyset$, hence $\bigcup_{i \in J} B_{i}$ is closed in $U$. Consider now a point $z \in \partial_{U}\left(\bigcup_{i \in J} B_{i}\right)$. Its neighbourhood $\bigcup_{i \in I(z)} B_{i}$ contains necessarily two or three bricks, (at least) one of which is in $\left\{B_{i}\right\}_{i \in J}$ and (at least) one is not. The result is then obvious by Fig. 5 .

Let $\mathcal{D}=\left\{B_{i}\right\}_{i \in I}$ be a brick decomposition of an open set $U \subset \mathbb{S}^{2}$ and let $h$ be a homeomorphism of $\mathbb{S}^{2}$ such that $h(U)=U$. For a given brick $B_{i_{0}} \in \mathcal{D}$, we recall the notions of attractor and repeller associated to $B_{i_{0}}$ (and $h$ ). We define

$$
I_{0}=\left\{i_{0}\right\}, \quad \mathcal{A}_{0}=\mathcal{R}_{0}=\bigcup_{i \in I_{0}} B_{i}=B_{i_{0}}
$$

and inductively, for $n \in \mathbb{N}$,

$$
\begin{array}{cc}
I_{n+1}=\left\{i \in I \mid h\left(\mathcal{A}_{n}\right) \cap B_{i} \neq \emptyset\right\}, & \mathcal{A}_{n+1}=\bigcup_{i \in I_{n+1}} B_{i}, \\
I_{-n-1}=\left\{i \in I \mid h^{-1}\left(\mathcal{R}_{-n}\right) \cap B_{i} \neq \emptyset\right\}, & \mathcal{R}_{-n-1}=\bigcup_{i \in I_{-n-1}} B_{i} .
\end{array}
$$

Definition 5.7. With the above notations, the sets

$$
\mathcal{A}=\bigcup_{n \geq 1} \mathcal{A}_{n}, \quad \mathcal{R}=\bigcup_{n \geq 1} \mathcal{R}_{-n}
$$

are called respectively the attractor and repeller associated to the brick $B_{i_{0}}$.

Note that according to Property 5.6, $\mathcal{A}$ and $\mathcal{R}$ are closed subsets of $U$. The proof of the following easy property is left to the reader.

Property 5.8. We have $h\left(\mathcal{A} \cup B_{i_{0}}\right) \subset \operatorname{Int}(\mathcal{A})$. Consequently, $h^{k}\left(\partial_{U} \mathcal{A}\right) \cap$ $h^{l}\left(\partial_{U} \mathcal{A}\right)=\emptyset$ for any two integers $k \neq l$.

We will use brick decompositions with a homeomorphism of $\mathbb{S}^{2}$ which reverses the orientation and without a 2-periodic point. The next result describes what are the "good" brick decompositions in this setting and then gives two essential properties for $\mathcal{A}$ and $\mathcal{R}$.

Lemma 5.9. Let $\mathcal{D}=\left\{B_{i}\right\}_{i \in I}$ be a brick decomposition of an open set $U \subset \mathbb{S}^{2}$ and let $h$ be an orientation reversing homeomorphism of $\mathbb{S}^{2}$ which has no 2-periodic point and satisfies $h(U)=U$. Assume furthermore that $\mathcal{D}$ satisfies the following two hypotheses: 
(H1) Every brick $B_{i}$ satisfies $B_{i} \cap h\left(B_{i}\right)=\emptyset=B_{i} \cap h^{2}\left(B_{i}\right)$,

(H2) For any two bricks $B_{i}, B_{j}$, at most one of the two sets $B_{i} \cap h\left(B_{j}\right)$ or $B_{i} \cap h^{-1}\left(B_{j}\right)$ is nonempty.

Then, for any brick $B_{i_{0}} \in \mathcal{D}$, the attractor $\mathcal{A}$ and the repeller $\mathcal{R}$ associated to $B_{i_{0}}$ are such that

(i) $\operatorname{Int}\left(B_{i_{0}}\right) \cap \mathcal{A}=\emptyset$,

(ii) $\mathcal{A} \cap \operatorname{Int}(\mathcal{R})=\emptyset$.

Proof. (i) Observe that $\operatorname{Int}\left(B_{i_{0}}\right) \cap \mathcal{A} \neq \emptyset$ simply means that there exist an integer $n \geq 1$ and a sequence of bricks $B_{i_{0}}, B_{i_{1}}, \ldots, B_{i_{n}}=B_{i_{0}}$ such that

$$
\forall k \in\{0, \ldots, n-1\} \quad h\left(B_{i_{k}}\right) \cap B_{i_{k+1}} \neq \emptyset .
$$

This contradicts Lemma 5.4.

(ii) If $\mathcal{A} \cap \operatorname{Int}(\mathcal{R}) \neq \emptyset$ then there exist two integers $n, m \geq 1$ and two sequences of bricks, say $B_{i_{0}}, B_{i_{1}}, \ldots, B_{i_{m}}$ and $B_{j_{0}}, B_{j_{1}}, \ldots, B_{j_{n}}$, such that

$$
\begin{aligned}
& B_{i_{0}}=B_{j_{0}}, \quad B_{i_{m}}=B_{j_{n}}, \\
& \forall k \in\{0, \ldots, m-1\} \quad h\left(B_{i_{k}}\right) \cap B_{i_{k+1}} \neq \emptyset, \\
& \forall l \in\{0, \ldots, n-1\} \quad h^{-1}\left(B_{j_{l}}\right) \cap B_{j_{l+1}} \neq \emptyset .
\end{aligned}
$$

As an immediate consequence, the $h$-image of each brick in the sequence

$$
B_{j_{n}}, \ldots, B_{j_{1}}, B_{j_{0}}=B_{i_{0}}, \ldots, B_{i_{m-1}}
$$

meets the next brick. This contradicts again Lemma 5.4 since $h\left(B_{i_{m-1}}\right)$ meets $B_{i_{m}}=B_{j_{n}}$.

5.3. Proof of Theorem 5.1. Let $h$ and $m$ be as in Theorem 5.1. We define $U=\mathbb{S}^{2} \backslash \operatorname{Fix}(h)=\mathbb{S}^{2} \backslash \operatorname{Fix}\left(h^{2}\right)$. Of course we have $h(U)=U \neq \emptyset$, and according to the Lefschetz-Hopf Theorem, $U \neq \mathbb{S}^{2}$. Let us remark that there is a situation where our result is easily seen. According to a theorem of Epstein, a connected component $K$ of $\operatorname{Fix}(h)$ is either a point or an arc or a Jordan curve, and in the last two cases, $h$ interchanges locally the two sides of $K$ : see [6, Theorem 2.5]. If one can choose $K$ to be a Jordan curve then $\mathbb{S}^{2} \backslash K$ has exactly two connected components, say $U_{1}$ and $U_{2}$ with $m \in U_{1}$, which are interchanged by $h$ (this also implies $K=\operatorname{Fix}(h)$ ). Since the $U_{i}$ 's are homeomorphic to $\mathbb{R}^{2}$ we can use the Brouwer plane translation theorem with $\left.h^{2}\right|_{U_{1}}$ to find a proper topological embedding $\varphi:\left\{(x, y) \in \mathbb{R}^{2} \mid y>0\right\} \rightarrow U_{1}$ such that $\varphi(0,1)=m$ and $h^{2} \circ \varphi(x, y)=\varphi \circ \tau(x, y)$ for $y>0$, where $\tau(x, y)=(x+2, y)=G^{2}(x, y)$. We obtain a proper topological embedding $\varphi: \mathcal{O}=\left\{(x, y) \in \mathbb{R}^{2} \mid y \neq 0\right\} \rightarrow U$ such that $h \circ \varphi=\left.\varphi \circ G\right|_{\mathcal{O}}$ by defining

$$
\forall y<0 \quad \varphi(x, y)=h \circ \varphi \circ G^{-1}(x, y) \in U_{2} .
$$

Thus we can suppose that $\mathbb{S}^{2} \backslash K$ is connected for every connected component $K$ of $\operatorname{Fix}(h)$, and this implies that $U=\mathbb{S}^{2} \backslash \operatorname{Fix}(h)$ is connected 
(see for example [18, Theorem 14.3, p. 123]). According to Lemma 2.8 and Propositions 3.2 and 3.7, at least one of the following two properties is true:

(P1) There exists a translation arc $\alpha$ for $h$ containing the point $m$ and such that $\bigcup_{k \in \mathbb{Z}} h^{k}(\alpha)$ is a simple curve contained in $U$.

(P2) There exists a translation arc $\beta$ for $h^{2}$ containing the point $m$ and such that $\bigcup_{k \in \mathbb{Z}} h^{2 k}(\beta)$ and $\bigcup_{k \in \mathbb{Z}} h^{2 k+1}(\beta)$ are two disjoint simple curves contained in $U$.

5.3.1. Proof when $(\mathrm{P} 1)$ is true. Up to conjugacy in $\mathbb{S}^{2}$, we can suppose that

$$
\begin{aligned}
h^{-1}(\alpha) & =[-1,0] \times\{0\}, \\
h(x, y) & =(x+1,-y) \text { for every }(x, y) \in h^{-1}(\alpha) \cup \alpha=[-1,1] \times\{0\}, \\
m & =(3 / 4,0) .
\end{aligned}
$$

For $\varepsilon>0$ we consider the three rectangles (see Fig. 7)

$$
\begin{aligned}
D_{-1} & =\left\{(x, y) \in \mathbb{R}^{2} \mid-1 / 4 \leq x \leq 1 / 4 \text { and }-\varepsilon \leq y \leq \varepsilon\right\}, \\
D_{0} & =\left\{(x, y) \in \mathbb{R}^{2} \mid 1 / 4 \leq x \leq 3 / 4 \text { and }-\varepsilon \leq y \leq \varepsilon\right\}, \\
D_{1} & =\left\{(x, y) \in \mathbb{R}^{2} \mid 3 / 4 \leq x \leq 5 / 4 \text { and }-\varepsilon \leq y \leq \varepsilon\right\} .
\end{aligned}
$$

Lemma 5.10 (adapted from [19]). There exist $\varepsilon>0$ and a brick decomposition $\mathcal{D}=\left\{B_{i}\right\}_{i \in \mathbb{N}}$ of $U$ such that:

1. $D_{-1}, D_{0}$ and $D_{1}$ are bricks of $\mathcal{D}$,

2. $\mathcal{D}$ satisfies hypotheses $(\mathrm{H} 1)$ and $(\mathrm{H} 2)$ in Lemma 5.9.

Proof. Clearly one can choose $\varepsilon>0$ so small that for $k, l$ in $\{0, \pm 1\}$ we have

- $D_{k} \cap h\left(D_{k}\right)=\emptyset=D_{k} \cap h^{2}\left(D_{k}\right)$,

- $D_{k} \cap h\left(D_{l}\right)=\emptyset$ or $D_{k} \cap h^{-1}\left(D_{l}\right)=\emptyset$.

Set $V=U \backslash \operatorname{Int}\left(D_{-1} \cup D_{0} \cup D_{1}\right)$. This is a noncompact bordered surface with only one boundary component, namely $\partial\left(D_{-1} \cup D_{0} \cup D_{1}\right)$, so there exists a countable triangulation $\mathcal{T}$ of $V$. Let $\mathcal{T}^{\prime}$ be (for example) the barycentric subdivision of $\mathcal{T}$. If the middle $p$ of some edge $E$ of $\mathcal{T}$ is a vertex of a rectangle $D_{k}(k=0, \pm 1)$, we slightly alter $\mathcal{T}^{\prime}$ by replacing $p$ with a close point $q \in E$. Note that this requires only finitely many modifications since there are only finitely many triangles of $\mathcal{T}$ meeting $\partial\left(D_{-1} \cup D_{0} \cup D_{1}\right)$. We continue to write $\mathcal{T}^{\prime}$ for this "perturbation" of $\mathcal{T}^{\prime}$. Then we define

$$
\mathcal{D}_{1}=\left\{D_{-1}, D_{0}, D_{1}\right\} \cup\left\{\operatorname{Star}\left(v, \mathcal{T}^{\prime}\right) \mid v \text { vertex of } \mathcal{T}\right\},
$$

where $\operatorname{Star}\left(v, \mathcal{T}^{\prime}\right)$ is the union of the triangles of $\mathcal{T}^{\prime}$ containing $v$. It is left to the reader to show that $\mathcal{D}_{1}$ is a brick decomposition of $U$ and in particular that, for any vertex $v$ of $\mathcal{T}$, the set $\operatorname{Star}\left(v, \mathcal{T}^{\prime}\right) \cap D_{k}$ is either empty or an arc $(k=0, \pm 1)$. If condition (H1) does not hold for $\mathcal{D}_{1}$, we can achieve 
it as follows: the bricks $B \in \mathcal{D}_{1}$ such that $B \cap\left(h(B) \cup h^{2}(B)\right) \neq \emptyset$ can be numbered $B_{1}, B_{2}, \ldots$ and $D_{0}, D_{ \pm 1}$ are not in this (finite or countable) sequence because of the choice of $\varepsilon$. Up to homeomorphism, we can suppose that $B_{1}$ is a rectangle. It can be subdivided into finitely many subrectangles $B_{1,1}, B_{1,2}, \ldots, B_{1, n_{1}}$ disjoint from their images under $h$ and $h^{2}$; it suffices that all these $B_{1, j}$ have diameter less than the infimum of $\mathrm{d}(z, h(z))$ and $\mathrm{d}\left(z, h^{2}(z)\right)$, for $z$ in $B_{1}$, where $\mathrm{d}(\cdot, \cdot)$ is any distance defining the topology of $\mathbb{S}^{2}$. Furthermore, since $B_{1}$ meets only finitely many bricks of $\mathcal{D}_{1}$, this can be done in such a way that we still have a brick decomposition of $U$ after replacing $B_{1}$ with $B_{1,1}, B_{1,2}, \ldots, B_{1, n_{1}}$ in $\mathcal{D}_{1}$ (see Fig. 6 ). We write $\mathcal{D}_{1,1}$ for this new brick decomposition of $U$. Now construct similarly a brick decomposition of $U$,

$$
\mathcal{D}_{1,2}=\left(\mathcal{D}_{1,1} \backslash\left\{B_{2}\right\}\right) \cup\left\{B_{2,1}, B_{2,2}, \ldots, B_{2, n_{2}}\right\},
$$

where $B_{2,1}, B_{2,2}, \ldots, B_{2, n_{2}}$ come from a suitable subdivision of $B_{2}$, and so on. Then $\mathcal{D}_{2}=\left(\mathcal{D}_{1} \backslash\left\{B_{1}, B_{2}, \ldots\right\}\right) \cup \bigcup_{i, j}\left\{B_{i, j}\right\}$ is a brick decomposition satisfying (H1) and having $D_{0}, D_{ \pm 1}$ as bricks.

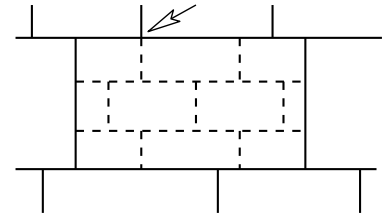

Not allowed

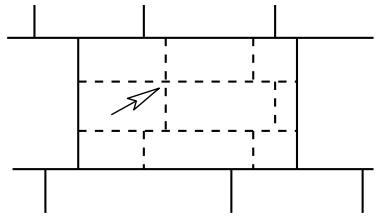

Not allowed

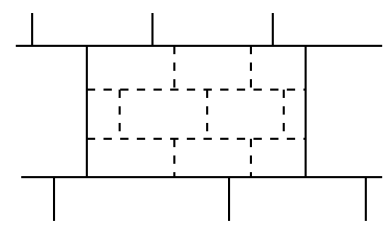

Allowed

Fig. 6. Subdivision of a brick

Suppose now that (H2) is not satisfied for $\mathcal{D}_{2}$, i.e. the set

$$
\left\{B \in \mathcal{D}_{2} \mid \exists B^{\prime} \in \mathcal{D}_{2} \text { such that } B \cap h^{-1}\left(B^{\prime}\right) \neq \emptyset \text { and } B \cap h\left(B^{\prime}\right) \neq \emptyset\right\}
$$

is nonempty. The bricks in the last set other than $D_{0}, D_{ \pm 1}$ are numbered $B_{1}, B_{2}, \ldots$. Since $h\left(B_{1}\right) \cup h^{-1}\left(B_{1}\right)$ is compact, there are only finitely many corresponding $B^{\prime}$, say $B_{1}^{\prime}, \ldots, B_{n}^{\prime}$. Each $B_{k}^{\prime}$ is disjoint from its image under $h^{2}$ so there exists $\delta>0$ such that

$$
\forall k \in\{1, \ldots, n\} \quad \operatorname{dist}\left(h^{-1}\left(B_{k}^{\prime}\right), h\left(B_{k}^{\prime}\right)\right)>\delta,
$$

where $\operatorname{dist}(X, Y)$ is the distance between two subsets $X, Y$ of $\mathbb{S}^{2}$. As above we can subdivide $B_{1}$ into "subbricks" with diameter less than $\delta$ and so carefully that we still have a brick decomposition of $U$, say $\mathcal{D}_{2,1}$, when $B_{1}$ is replaced with its subbricks in $\mathcal{D}_{2}$. Afterwards, define $\mathcal{D}_{2,2}$ by replacing $B_{2}$ with suitable subbricks in $\mathcal{D}_{2,1}$, etc. It is easily seen that replacing in $\mathcal{D}_{2}$ the bricks $B_{1}, B_{2}, \ldots$ with their subbricks, we obtain a brick decomposition of $U$ which satisfies hypotheses (H1), (H2) and has $D_{0}, D_{ \pm 1}$ as bricks. 
Consider the attractor $\mathcal{A}$ and the repeller $\mathcal{R}$ associated to $B_{i_{0}}=D_{0}$. We remark that

$$
D_{1} \subset \mathcal{A}_{1} \subset \mathcal{A}, \quad D_{-1} \subset \mathcal{R}_{-1} \subset \mathcal{R},
$$

since respectively $(5 / 4,0) \in h\left(D_{0}\right) \cap D_{1}$ and $(-1 / 4,0) \in h^{-1}\left(D_{0}\right) \cap D_{-1}$.

According to Lemma 5.9(i), the vertical segment $\{3 / 4\} \times[-\varepsilon, \varepsilon]$ is contained in a connected component $\Delta$ of $\partial_{U} \mathcal{A}$. Furthermore we know from Property 5.6 that $\Delta$ is either a Jordan curve or is homeomorphic to $\mathbb{R}$. Before dealing with these two situations, we give the following notations and an elementary but important lemma.

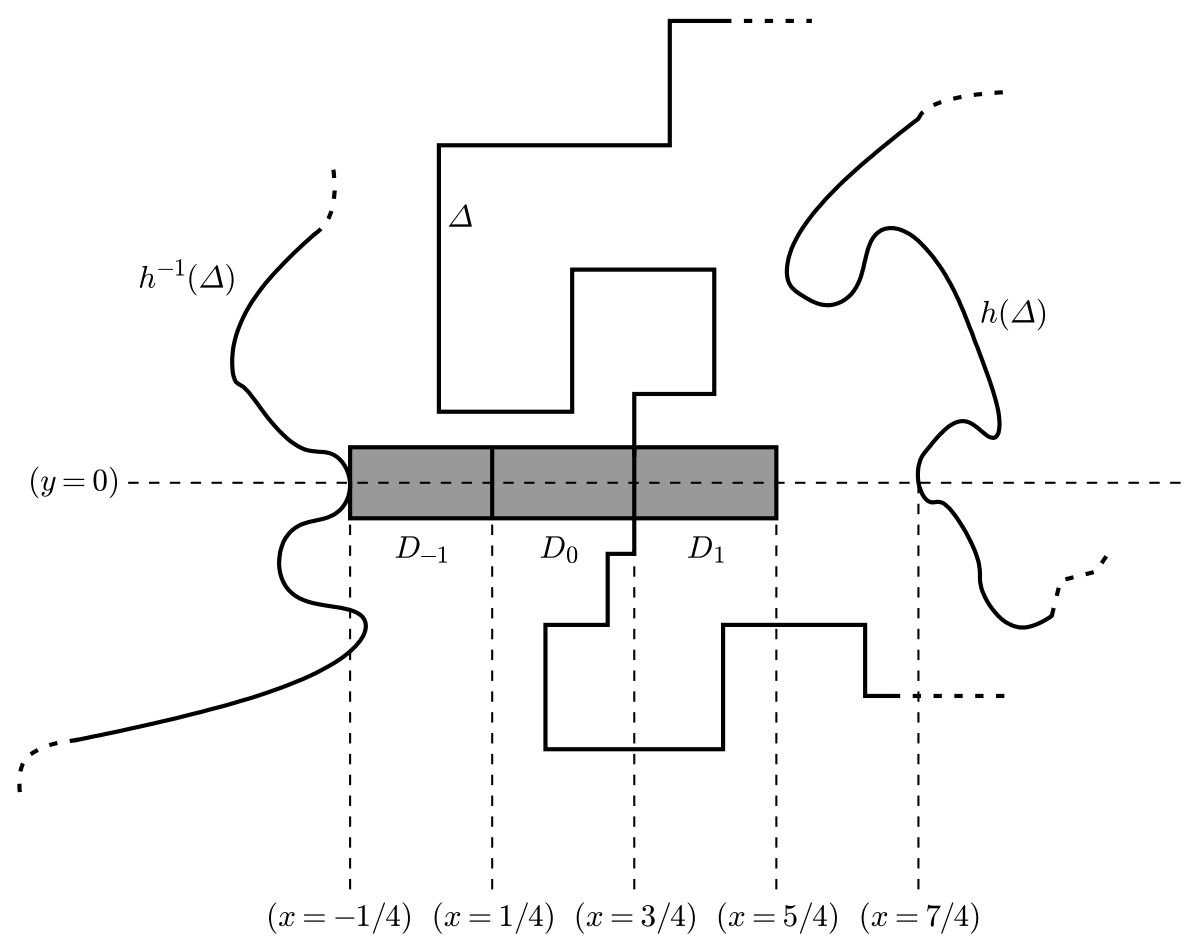

Fig. 7. The bricks $D_{0}, D_{ \pm 1}$ and $\Delta, h^{ \pm 1}(\Delta)$ close to these bricks

Notations 5.11.

$$
\begin{aligned}
\gamma_{-} & =\{(x, 0) \mid-1 / 4<x<3 / 4\}, \\
\gamma_{+} & =\{(x, 0) \mid 3 / 4<x<7 / 4\}=h\left(\gamma_{-}\right), \\
\gamma & =\{(x, 0) \mid-1 / 4<x<7 / 4\}=\gamma_{-} \cup\{(3 / 4,0)\} \cup \gamma_{+} .
\end{aligned}
$$

Lemma 5.12. The set $h^{-1}(\Delta) \cup \gamma_{-}\left(\right.$resp. $\left.\gamma_{+} \cup h(\Delta)\right)$ is connected and contained in $U \backslash \mathcal{A}($ resp. in $\operatorname{Int}(\mathcal{A}))$. 
Proof. These sets are obviously contained in $U$. For the connectedness, it is enough to remark that

$$
(-1 / 4,0) \in h^{-1}(\Delta) \cap \mathrm{Cl}\left(\gamma_{-}\right), \quad(7 / 4,0) \in \mathrm{Cl}\left(\gamma_{+}\right) \cap h(\Delta) .
$$

Property 5.8 gives $h(\Delta) \subset h(\mathcal{A}) \subset \operatorname{Int}(\mathcal{A})$ and also

$$
h^{-1}(\Delta) \cap \mathcal{A}=h^{-1}(\Delta \cap h(\mathcal{A})) \subset h^{-1}\left(\partial_{U} \mathcal{A} \cap \operatorname{Int}(\mathcal{A})\right)=\emptyset .
$$

According to Lemma 5.9 we have

$$
\operatorname{Int}\left(D_{-1}\right) \cap \mathcal{A} \subset \operatorname{Int}(\mathcal{R}) \cap \mathcal{A}=\emptyset, \quad \operatorname{Int}\left(D_{0}\right) \cap \mathcal{A}=\emptyset,
$$

hence $\gamma_{-} \cap \mathcal{A}=\emptyset$.

It remains to check that $\gamma_{+} \subset \operatorname{Int}(\mathcal{A})$. This follows from

$$
\{(x, 0) \mid 3 / 4<x<5 / 4\} \subset \operatorname{Int}\left(D_{1}\right) \subset \operatorname{Int}(\mathcal{A})
$$

and, by Property 5.8, from

$$
\{(x, 0) \mid 5 / 4 \leq x<7 / 4\} \subset h\left(D_{0}\right) \subset \operatorname{Int}(\mathcal{A}) .
$$

CASE 1. The set $\Delta$ is a Jordan curve.

Claim 1. The set $\Delta$ separates $h^{-1}(\Delta)$ and $h(\Delta)$ in $\mathbb{S}^{2}$.

Proof. If this were not true, Lemma 5.12 would show that the segment $\gamma$ intersects $\Delta$ transversely and meets only one connected component of $\mathbb{S}^{2} \backslash \Delta$, which is absurd.

Let us write $V_{+}$for the connected component of $\mathbb{S}^{2} \backslash \Delta$ containing $h(\Delta)$. We have $\partial h\left(V_{+}\right)=h(\Delta) \subset V_{+}$so $h\left(V_{+}\right) \cap V_{+} \neq \emptyset$ and actually $h\left(\mathrm{Cl}\left(V_{+}\right)\right)$ $\subset V_{+}$, since according to the above claim,

$$
h\left(V_{+}\right) \cap \partial V_{+}=h\left(V_{+}\right) \cap \Delta=h\left(V_{+} \cap h^{-1}(\Delta)\right)=\emptyset .
$$

It is now routine to construct a topological embedding $\varphi$ defined on $\mathcal{O}=$ $\mathbb{R}^{2} \backslash\{(0,0)\}$ and conjugating $h$ and $H$. We just sketch the construction: defining $\Omega=V_{+} \backslash h\left(\mathrm{Cl}\left(V_{+}\right)\right)$, we clearly have $\mathrm{Cl}(\Omega)=\Delta \cup \Omega \cup h(\Delta) \subset U$. Let $\varphi: \mathbb{S}^{1} \rightarrow \Delta$ be a homeomorphism. It can be extended to a homeomorphism

$$
\varphi: \mathbb{S}^{1} \cup H\left(\mathbb{S}^{1}\right) \rightarrow \Delta \cup h(\Delta)
$$

by defining $\left.\varphi\right|_{H\left(\mathbb{S}^{1}\right)}=\left.h \circ \varphi \circ H^{-1}\right|_{H\left(\mathbb{S}^{1}\right)}$. Using suitably the Schoenflies Theorem, one can extend again $\varphi$ to a homeomorphism from the compact annulus $A=\{z \in \mathbb{C}|1 / 2 \leq| z \mid \leq 1\}$ onto $\mathrm{Cl}(\Omega)$. Finally, for any point $z \in \mathbb{R}^{2} \backslash\{(0,0)\}$, there exists a unique $k \in \mathbb{Z}$ such that $z \in H^{k}\left(A \backslash \partial^{-} A\right)$, where $\partial^{-} A=\{z \in \mathbb{C}|| z \mid=1 / 2\}$, and we define

$$
\varphi(z)=h^{k} \circ \varphi \circ H^{-k}(z) \in h^{k}(\mathrm{Cl}(\Omega)) .
$$

One can easily check that $\varphi: \mathcal{O}=\mathbb{R}^{2} \backslash\{(0,0)\} \rightarrow U$ is a well-defined one-toone continuous map such that $h \circ \varphi=\left.\varphi \circ H\right|_{\mathcal{O}}$ and $\varphi(\mathcal{O})=\bigcup_{k \in \mathbb{Z}} h^{k}(\mathrm{Cl}(\Omega))$. 
CASE 2. The set $\Delta$ is homeomorphic to $\mathbb{R}$. Since $\Delta$ is a closed subset of $U$ we have

$$
\emptyset \neq \operatorname{Cl}(\Delta) \backslash \Delta \subset \operatorname{Fix}(h) .
$$

Moreover, $\operatorname{Cl}(\Delta) \backslash \Delta$ has at most two connected components, say $L_{1}$ and $L_{2}$ with possibly $L_{1}=L_{2}$, and each $L_{i}$ is contained in a connected component $K_{i}$ of $\operatorname{Fix}(h)$. It will be convenient to compactify $\mathbb{S}^{2} \backslash\left(K_{1} \cup K_{2}\right)$ as follows: choose $a_{1}$ and $a_{2}$ in $\mathbb{S}^{2}$ with the convention that $a_{1}=a_{2}$ if and only if $K_{1}=K_{2}$. Since $U$ has been assumed to be connected, so is $\mathbb{S}^{2} \backslash\left(K_{1} \cup K_{2}\right)$ and it is then very classical that this last set is homeomorphic to $\mathbb{S}^{2} \backslash\left\{a_{1}, a_{2}\right\}$ (see for example [18, Chapter VI]). Now, if $\psi$ is any homeomorphism from $\mathbb{S}^{2} \backslash\left(K_{1} \cup K_{2}\right)$ onto $\mathbb{S}^{2} \backslash\left\{a_{1}, a_{2}\right\}$, we define $\widehat{h}: \mathbb{S}^{2} \rightarrow \mathbb{S}^{2}$ by

$$
\widehat{h}(z)= \begin{cases}z & \text { if } z \in\left\{a_{1}, a_{2}\right\}, \\ \psi \circ h \circ \psi^{-1}(z) & \text { if } z \notin\left\{a_{1}, a_{2}\right\} .\end{cases}
$$

One can check that $\widehat{h}$ is a homeomorphism and that $\operatorname{Cl}(\psi(\Delta)) \backslash \psi(\Delta)=$ $\left\{a_{1}, a_{2}\right\}$. Furthermore, since we are looking for a (proper) topological embedding $\varphi$ with values in $U \subset \mathbb{S}^{2} \backslash\left(K_{1} \cup K_{2}\right)$, it is enough to prove our theorem for $\widehat{h}$ instead of $h$. In other words, there is no loss in supposing that $K_{i}$ (and so $L_{i}$ ) is one point $(i \in\{1,2\})$. This will be assumed from now on.

Claim 2. We have necessarily $K_{1}=K_{2}$.

Proof. Suppose this is not true and define

$$
C=\mathrm{Cl}(\Delta \cup h(\Delta))=\Delta \cup h(\Delta) \cup K_{1} \cup K_{2} .
$$

Thus $C$ is a Jordan curve. The sets $h^{-1}(\Delta) \cup \gamma_{-}$and $\gamma_{+}$are both connected and contained in $U \backslash(\Delta \cup h(\Delta)) \subset \mathbb{S}^{2} \backslash C$ : for $h^{-1}(\Delta) \cup \gamma_{-}$, this is contained in Lemma 5.12 since we know from Property 5.8 that $\Delta \cup h(\Delta) \subset \mathcal{A}$. Lemma 5.12 also gives

$$
\gamma_{+} \cap \Delta \subset \operatorname{Int}(\mathcal{A}) \cap \partial_{U} \mathcal{A}=\emptyset
$$

and

$$
\gamma_{+} \cap h(\Delta)=h\left(\gamma_{-} \cap \Delta\right) \subset h\left(\gamma_{-} \cap \mathcal{A}\right)=\emptyset .
$$

Now, since the segment $\gamma$ intersects $\Delta \subset C$ transversely, we deduce that the connected components $V_{-}, V_{+}$of $\mathbb{S}^{2} \backslash C$ containing respectively $h^{-1}(\Delta) \cup \gamma_{-}$ and $\gamma_{+}$are different. It follows that

$$
\partial h^{-1}\left(V_{+}\right) \cap V_{+}=h^{-1}(C) \cap V_{+}=h^{-1}(\Delta) \cap V_{+}=\emptyset
$$

so either $V_{+} \subset h^{-1}\left(V_{+}\right)$or $V_{+} \cap h^{-1}\left(V_{+}\right)=\emptyset$. We now remark that none of these two situations is possible. The first one would imply

$$
\gamma_{+} \cup \gamma_{-}=\gamma_{+} \cup h^{-1}\left(\gamma_{+}\right) \subset h^{-1}\left(V_{+}\right),
$$




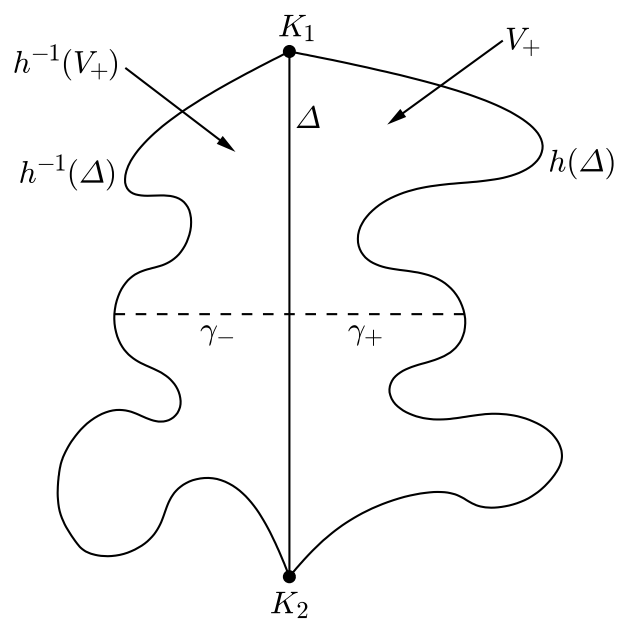

Fig. 8. $V_{+} \cap h^{-1}\left(V_{+}\right)=\emptyset$ is not possible

which is absurd because the segment $\gamma$ intersects $\Delta \subset h^{-1}(C)$ transversely. Suppose now that $h^{-1}\left(V_{+}\right) \cap V_{+}=\emptyset$. We cannot have $h^{-1}\left(\mathrm{Cl}\left(V_{+}\right)\right) \cup \mathrm{Cl}\left(V_{+}\right)$ $=\mathbb{S}^{2}$ since this would imply $h^{-1}(\Delta)=h(\Delta)$, which contradicts Property 5.8. So $h^{-1}\left(\mathrm{Cl}\left(V_{+}\right)\right) \cup \mathrm{Cl}\left(V_{+}\right)$is contained in the domain of a single chart of $\mathbb{S}^{2}$ and can be represented as in Fig. 8. Keeping in mind that $K_{1}, K_{2}$ are fixed points of $h$, this contradicts the fact that $h$ reverses the orientation.

Thus $\mathrm{Cl}(\Delta)=\Delta \cup K_{1}$ is a Jordan curve. Again, $\gamma$ intersects $\Delta \subset \mathrm{Cl}(\Delta)$ transversely so we conclude from Lemma 5.12:

Claim 3. The set $\mathrm{Cl}(\Delta)$ separates $h^{-1}(\Delta)$ and $h(\Delta)$ in $\mathbb{S}^{2}$.

Now, let $V_{+}$be the connected component of $\mathbb{S}^{2} \backslash \mathrm{Cl}(\Delta)$ containing $h(\Delta)$. Since $h(\Delta) \subset \partial h\left(V_{+}\right) \cap V_{+}$we have $h\left(V_{+}\right) \cap V_{+} \neq \emptyset$ and in fact $h\left(V_{+} \cup \Delta\right) \subset V_{+}$ because Claim 3 implies

$$
h\left(V_{+}\right) \cap \partial V_{+}=h\left(V_{+}\right) \cap \mathrm{Cl}(\Delta)=h\left(V_{+}\right) \cap \Delta=h\left(V_{+} \cap h^{-1}(\Delta)\right)=\emptyset .
$$

We conclude as follows. Define $\Omega=V_{+} \backslash h\left(\mathrm{Cl}\left(V_{+}\right)\right)$. Obviously $\mathrm{Cl}(\Omega) \backslash K_{1}=$ $\Delta \cup \Omega \cup h(\Delta) \subset U$. Using the Schoenflies Theorem, one can construct a homeomorphism

$$
\varphi:\left\{(x, y) \in \mathbb{R}^{2} \mid 0 \leq x \leq 1\right\} \cup\{\infty\} \rightarrow \mathrm{Cl}(\Omega)
$$

such that $\varphi(\infty)=K_{1}, \varphi(\{0\} \times \mathbb{R})=\Delta$ and

$$
\forall y \in \mathbb{R} \quad \varphi(1, y)=h \circ \varphi \circ G^{-1}(1, y) \in h(\Delta) .
$$

Now, if $k \leq x<k+1(k \in \mathbb{Z})$ we let

$$
\varphi(x, y)=h^{k} \circ \varphi \circ G^{-k}(x, y) \in h^{k}(\Delta \cup \Omega) .
$$


It is easily seen that $\varphi: \mathcal{O}=\mathbb{R}^{2} \rightarrow U$ defined in this way is a proper topological embedding, with image $\varphi(\mathcal{O})=\bigcup_{k \in \mathbb{Z}} h^{k}(\Delta \cup \Omega)$, such that $h \circ \varphi$ $=\left.\varphi \circ G\right|_{\mathcal{O}}$. This completes the proof of Theorem 5.1 when Property (P1) is true.

5.3.2. Proof when $(\mathrm{P} 2)$ is true. Up to conjugacy in $\mathbb{S}^{2}$, we can suppose that

$$
\begin{aligned}
& h^{-2}(\beta)=[-2,0] \times\{-1\}, \\
& h(x, y)=(x+1,-y) \text { if }(x, y) \in \bigcup_{k=-2}^{1} h^{k}(\beta)=[-2,2] \times\{-1\} \cup[-1,3] \times\{1\}, \\
& m=(3 / 2,-1) .
\end{aligned}
$$

For $\varepsilon>0$, consider the five rectangles (see Fig. 9)

$$
D_{i}=\left\{\begin{array}{c}
\{(x, y) \mid(i+1) / 2 \leq x \leq(i+3) / 2 \text { and }-1-\varepsilon \leq y \leq-1+\varepsilon\} \\
\text { for } i \in\{0, \pm 2\}, \\
\{(x, y) \mid(i+1) / 2 \leq x \leq(i+3) / 2 \text { and } 1-\varepsilon \leq y \leq 1+\varepsilon\} \\
\text { for } i= \pm 1 .
\end{array}\right.
$$

The proof of the next lemma is similar to the one of Lemma 5.10 and will be omitted.

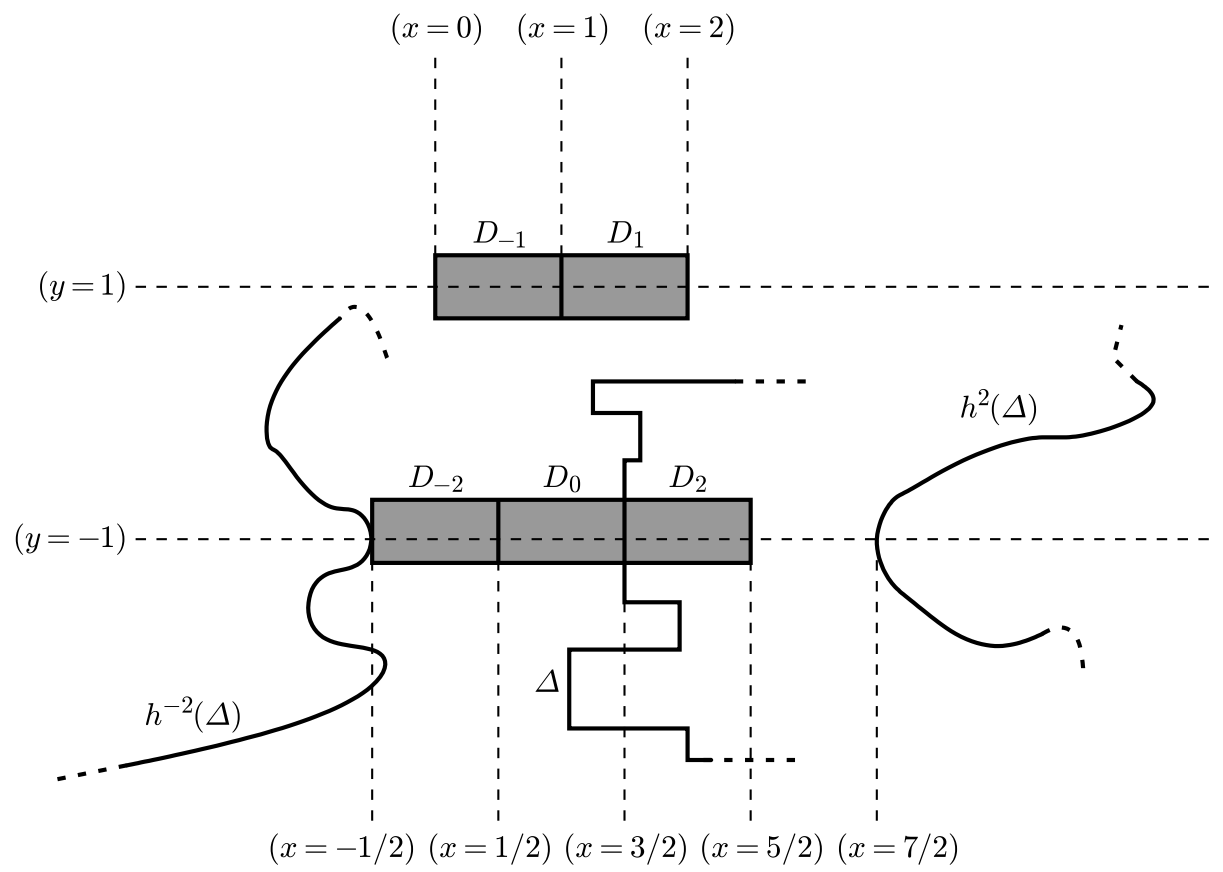

Fig. 9. The bricks $D_{0}, D_{ \pm 1}, D_{ \pm 2}$ and $\Delta, h^{ \pm 2}(\Delta)$ close to these bricks 
Lemma 5.13. There exist $\varepsilon>0$ and a brick decomposition $\mathcal{D}=\left\{B_{i}\right\}_{i \in \mathbb{N}}$ of $U$ such that:

1. $D_{0}, D_{ \pm 1}$ and $D_{ \pm 2}$ are bricks of $\mathcal{D}$,

2. $\mathcal{D}$ satisfies hypotheses (H1) and (H2) in Lemma 5.9.

We consider the attractor $\mathcal{A}$ and the repeller $\mathcal{R}$ associated to the brick $B_{i_{0}}=D_{0}$. First we remark that

$$
D_{1} \cup D_{2} \subset \mathcal{A}, \quad D_{-1} \cup D_{-2} \subset \mathcal{R},
$$

since, on the one hand, $(2,1) \in h\left(D_{0}\right) \cap D_{1}$ and $(2,-1) \in h\left(D_{1}\right) \cap D_{2}$, and on the other hand, $(0,1) \in h^{-1}\left(D_{0}\right) \cap D_{-1}$ and $(0,-1) \in h^{-1}\left(D_{-1}\right) \cap D_{-2}$. Using Lemma 5.9 , we see that the vertical segment $\{3 / 2\} \times[-1-\varepsilon,-1+\varepsilon]$ is contained in a connected component $\Delta$ of $\partial_{U} \mathcal{A}$. As in Section 5.3.1 we give some convenient notations and a basic lemma before studying the situation when $\Delta$ is homeomorphic to $\mathbb{S}^{1}$ (resp. to $\mathbb{R}$ ).

Notations 5.14.

$$
\begin{aligned}
\gamma_{-} & =\{(x,-1) \mid-1 / 2<x<3 / 2\}, \\
\gamma_{+} & =\{(x,-1) \mid 3 / 2<x<7 / 2\}=h^{2}\left(\gamma_{-}\right), \\
\gamma & =\{(x,-1) \mid-1 / 2<x<7 / 2\}=\gamma_{-} \cup\{(3 / 2,-1)\} \cup \gamma_{+} .
\end{aligned}
$$

Lemma 5.15. The set $h^{-2}(\Delta) \cup \gamma_{-}\left(\right.$resp. $\left.\gamma_{+} \cup h^{2}(\Delta)\right)$ is connected and contained in $U \backslash \mathcal{A}($ resp. in $\operatorname{Int}(\mathcal{A}))$.

The proof is similar to the one of Lemma 5.12 and is left to the reader.

CASE 1 . The set $\Delta$ is a Jordan curve.

Claim 4. The set $\Delta$ separates $h^{-1}(\Delta)$ and $h(\Delta)$ in $\mathbb{S}^{2}$.

Proof. First we remark that $\Delta$ separates $h^{-2}(\Delta)$ and $h^{2}(\Delta)$ in $\mathbb{S}^{2}$ : this follows from Lemma 5.15 and from the fact that $\gamma$ intersects $\Delta$ transversely. Denote by $V_{-}, V_{+}$the connected components of $\mathbb{S}^{2} \backslash \Delta$ containing respectively $h^{-2}(\Delta)$ and $h^{2}(\Delta)$. As in Section 5.3.1 (with $h^{2}$ in place of $h$ ), one can check that $h^{2}\left(\mathrm{Cl}\left(V_{+}\right)\right) \subset V_{+}$or equivalently $\mathrm{Cl}\left(V_{-}\right) \subset h^{2}\left(V_{-}\right)$. According to the Brouwer fixed point theorem, $h^{2}$ has fixed points $z_{-} \in V_{-}$and $z_{+} \in V_{+}$, which are also fixed points of $h$ since $h$ has no 2-periodic point. In particular,

$$
V_{+} \cap h\left(V_{+}\right) \neq \emptyset \neq V_{-} \cap h^{-1}\left(V_{-}\right) .
$$

We now deduce from $h(\Delta) \cap \Delta=\emptyset$ that $h(\Delta) \subset V_{+}$: otherwise we would have $h(\Delta) \subset V_{-}$and consequently

$$
V_{+} \cap \partial h\left(V_{+}\right)=V_{+} \cap h(\Delta)=\emptyset
$$

so $V_{+} \subset h\left(V_{+}\right) \subset h^{2}\left(V_{+}\right)$, which contradicts $h^{2}\left(\mathrm{Cl}\left(V_{+}\right)\right) \subset V_{+}$. We get similarly $h^{-1}(\Delta) \subset V_{-}$upon replacing $h, V_{+}$with $h^{-1}, V_{-}$. 
Defining $\Omega=V_{+} \backslash h\left(\mathrm{Cl}\left(V_{+}\right)\right)$, we now proceed exactly as in Section 5.3.1 to construct a topological embedding

$$
\varphi: \mathcal{O}=\mathbb{R}^{2} \backslash\{(0,0)\} \rightarrow U
$$

with image $\varphi(\mathcal{O})=\bigcup_{k \in \mathbb{Z}} h^{k}(\mathrm{Cl}(\Omega))$ such that $h \circ \varphi=\left.\varphi \circ H\right|_{\mathcal{O}}$.

CASE 2. The set $\Delta$ is homeomorphic to $\mathbb{R}$. We denote again by $L_{1}, L_{2}$ the connected components of the nonempty set $\mathrm{Cl}(\Delta) \backslash \Delta \subset \mathrm{Fix}(h)$, with possibly $L_{1}=L_{2}$. Each $L_{i}$ is contained in a connected component $K_{i}$ of $\operatorname{Fix}(h)$, and as explained in Section 5.3.1, there is no loss in supposing that $K_{i}$ (and so $L_{i}$ ) is one point.

For convenience we will use the following notations for the two half-planes on both sides of the $x$-axis:

$$
\begin{gathered}
P_{+}=\left\{(x, y) \in \mathbb{R}^{2} \mid y>0\right\}, \quad P_{-}=\left\{(x, y) \in \mathbb{R}^{2} \mid y<0\right\} . \\
\text { We first suppose } K_{1}=K_{2} .
\end{gathered}
$$

Then $\operatorname{Cl}(\Delta)=\Delta \cup K_{1}$ is a Jordan curve. Using again Lemma 5.15 and since $\gamma \cap \Delta$ is a transverse intersection, one deduces:

Claim 5. The set $\mathrm{Cl}(\Delta)$ separates $h^{-2}(\Delta)$ and $h^{2}(\Delta)$ in $\mathbb{S}^{2}$.

We now consider the two connected components $V_{-}, V_{+}$of $\mathbb{S}^{2} \backslash \mathrm{Cl}(\Delta)$, with $h^{2}(\Delta) \subset V_{+}$and $h^{-2}(\Delta) \subset V_{-}$. One can easily derive from the claim above that $h^{2}\left(V_{+} \cup \Delta\right) \subset V_{+}$, i.e. $V_{-} \cup \Delta \subset h^{2}\left(V_{-}\right)$.

Claim 6. There are three possible situations:

$$
\begin{aligned}
& h\left(V_{+} \cup \Delta\right) \subset V_{+}, \\
& h\left(V_{+} \cup \Delta\right) \subset V_{-}, \\
& h\left(V_{-} \cup \Delta\right) \subset V_{+} .
\end{aligned}
$$

Proof. Suppose that we are neither in situation (S1) nor (S2). Then $h\left(V_{+} \cup \Delta\right)$ meets $\partial V_{+}=\partial V_{-}=\operatorname{Cl}(\Delta)$. Since $h(\Delta) \cap \Delta=\emptyset$, this implies $h\left(V_{+}\right) \cap \Delta \neq \emptyset$ and then $\Delta \subset h\left(V_{+}\right)$. Consequently, $h\left(V_{-} \cup \Delta\right)$ is a connected subset of $\mathbb{S}^{2} \backslash \mathrm{Cl}(\Delta)$ and we get either $h\left(V_{-} \cup \Delta\right) \subset V_{+}$or $h\left(V_{-} \cup \Delta\right) \subset V_{-}$. The latter is actually impossible because $V_{-} \cup \Delta \subset h^{2}\left(V_{-}\right)$.

We now construct a proper topological embedding $\varphi: \mathcal{O} \rightarrow U$ conjugating $h$ and $G$ which will be defined on $\mathcal{O}=\mathbb{R}^{2}$ in the first situation and on $\mathcal{O}=\left\{(x, y) \in \mathbb{R}^{2} \mid y \neq 0\right\}$ in the last two.

- In situation (S1) we proceed exactly as in Section 5.3.1.

- Observe now that

$$
h\left(V_{-} \cup \Delta\right) \subset V_{+} \Leftrightarrow V_{-} \cup \Delta \subset h\left(V_{+}\right) \Leftrightarrow h^{-1}\left(V_{-} \cup \Delta\right) \subset V_{+},
$$

which shows that (S3) reduces to (S2) upon replacing $h$ with $h^{-1}$. Since it is equivalent to prove Theorem 5.1 for $h$ or for $h^{-1}$, it suffices to consider (S2). In this case, set $\Omega=V_{+} \backslash h^{2}\left(\mathrm{Cl}\left(V_{+}\right)\right)$. We then have $\mathrm{Cl}(\Omega) \backslash K_{1}=\Delta \cup \Omega \cup$ 
$h^{2}(\Delta) \subset U$. We construct the required embedding $\varphi$ as follows. We consider for example the set $D=\{(x, 1 / x) \mid x>0\}$ and we write $B$ for the domain between $D$ and $G^{2}(D)$ in the upper half-plane $P_{+}$. Using the Schoenflies Theorem, one can construct a homeomorphism

$$
\varphi: \mathrm{Cl}(B)=\mathrm{Cl}_{\mathbb{R}^{2}}(B) \cup\{\infty\} \rightarrow \mathrm{Cl}(\Omega)
$$

such that $\varphi(\infty)=K_{1}, \varphi(D)=\Delta$ and $\left.\varphi \circ G^{2}\right|_{D}=\left.h^{2} \circ \varphi\right|_{D}$. Then we define the map $\varphi$ on the half-plane $P_{+}$by observing that for every $z \in P_{+}$there exists a unique even integer $2 k$ such that $z \in G^{2 k}(D \cup B)$, and then defining

$$
\varphi(z)=h^{2 k} \circ \varphi \circ G^{-2 k}(z) \in h^{2 k}(\Delta \cup \Omega) .
$$

In particular, at this stage we have

$$
h^{2} \circ \varphi=\left.\varphi \circ G^{2}\right|_{P_{+}} .
$$

Next we extend $\varphi$ on $P_{-}$by

$$
\forall y<0 \quad \varphi(x, y)=h \circ \varphi \circ G^{-1}(x, y) \in \bigcup_{k \in \mathbb{Z}} h^{2 k+1}(\Delta \cup \Omega) .
$$

It is easily seen that in this way we have obtained a continuous map

$$
\varphi: \mathcal{O}=\left\{(x, y) \in \mathbb{R}^{2} \mid y \neq 0\right\} \rightarrow U
$$

satisfying $h \circ \varphi=\left.\varphi \circ G\right|_{\mathcal{O}}$ and such that, for every $x \in \mathbb{R}, \varphi((\{x\} \times \mathbb{R}) \cap \mathcal{O})$ is a closed subset of $U$. It is not entirely obvious that this map $\varphi$ is one-to-one (in contrast to the previously constructed embeddings). To check this property, it is enough to see that the sets $h^{k}(\Delta \cup \Omega), k \in \mathbb{Z}$, are pairwise disjoint. This turns out to be true because $h^{k}(\Delta) \cap h^{l}(\Delta)=\emptyset$ for $k \neq l$ (Property 5.8 ) and because

$$
\Omega \cap h(\Omega) \subset V_{+} \cap h\left(V_{+}\right)=\emptyset, \quad \Omega \cap h^{2}(\Omega) \subset \Omega \cap h^{2}\left(V_{+}\right)=\emptyset,
$$

which, according to Lemma 5.2, implies $h^{k}(\Omega) \cap h^{l}(\Omega)=\emptyset$ for $k \neq l$.

We now suppose $K_{1} \neq K_{2}$.

Define $C=\operatorname{Cl}\left(\Delta \cup h^{2}(\Delta)\right)=\Delta \cup h^{2}(\Delta) \cup K_{1} \cup K_{2}$. Thus $C$ is a Jordan curve.

Claim 7. The set $C$ separates $h^{-2}(\Delta)$ and $\gamma_{+}$in $\mathbb{S}^{2}$.

Proof. Property 5.8 gives $\Delta \cup h^{2}(\Delta) \subset \mathcal{A}$, so by Lemma $5.15, h^{-2}(\Delta) \cup \gamma_{-}$ is contained in a connected component $V_{-}$of $\mathbb{S}^{2} \backslash C$. This lemma also gives $\gamma_{+} \cap \Delta \subset \gamma_{+} \cap \partial_{U} \mathcal{A}=\emptyset$ and $\gamma_{+} \cap h^{2}(\Delta) \subset h^{2}\left(\gamma_{-} \cap \mathcal{A}\right)=\emptyset$, hence $\gamma_{+}$is also contained in a connected component $V_{+}$of $\mathbb{S}^{2} \backslash C$. We have necessarily $V_{-} \neq V_{+}$since the segment $\gamma$ intersects $\Delta \subset C$ transversely.

We keep the notations $V_{-}, V_{+}$of the proof above, that is, $V_{-}$(resp. $V_{+}$) is the connected component of $\mathbb{S}^{2} \backslash C$ containing $h^{-2}(\Delta)$ (resp. $\left.\gamma_{+}\right)$. In particular, $\partial V_{-}=\partial V_{+}=C$. 
Claim 8. We have $h^{2}\left(V_{+}\right) \cap V_{+}=\emptyset=h\left(V_{+}\right) \cap V_{+}$.

Proof. According to the previous claim we have

$$
\partial h^{-2}\left(V_{+}\right) \cap V_{+}=\left(h^{-2}(\Delta) \cup \Delta\right) \cap V_{+}=\emptyset
$$

so we have either $h^{-2}\left(V_{+}\right) \cap V_{+}=\emptyset$ or $V_{+} \subset h^{-2}\left(V_{+}\right)$. The latter would imply that $\gamma$ is contained in $h^{-2}\left(V_{+}\right)$except for the point $(3 / 2,-1)$, which is absurd since this segment intersects $\Delta \subset h^{-2}(C)$ transversely. This proves $h^{2}\left(V_{+}\right) \cap V_{+}=\emptyset$. For the other equality, we first observe that the situations $h^{ \pm 1}\left(V_{+}\right) \subset V_{+}$are not possible since they contradict $h^{2}\left(V_{+}\right) \cap V_{+}=\emptyset$. Suppose now $V_{+} \cap h\left(V_{+}\right) \neq \emptyset$. Then

$$
h\left(V_{+}\right) \cap C \neq \emptyset, \quad V_{+} \cap h(C) \neq \emptyset,
$$

that is,

$$
h\left(V_{+}\right) \cap\left(\Delta \cup h^{2}(\Delta)\right) \neq \emptyset, \quad V_{+} \cap\left(h(\Delta) \cup h^{3}(\Delta)\right) \neq \emptyset .
$$

For convenience we define

$$
E_{1}=h\left(V_{+}\right) \cap \Delta, E_{2}=h\left(V_{+}\right) \cap h^{2}(\Delta), E_{3}=V_{+} \cap h(\Delta), E_{4}=V_{+} \cap h^{3}(\Delta) .
$$

Since $h^{k}(\Delta) \cap h^{l}(\Delta)=\emptyset$ for $k \neq l$ we see that $E_{i}$ is either empty or equal, for $i=1,2,3,4$, respectively to the whole set $\Delta, h^{2}(\Delta), h(\Delta), h^{3}(\Delta)$.

It turns out that necessarily $E_{1}=\emptyset$, hence $E_{2}=h^{2}(\Delta)$. Otherwise we would have $\Delta \subset h\left(V_{+}\right)$, i.e. $h^{-1}(\Delta) \subset V_{+}$, and $h^{-1}(\mathrm{Cl}(\Delta))$ would be a connected set joining $K_{1}$ and $K_{2}$ in $\mathrm{Cl}\left(V_{+}\right)$. Moreover, $\mathrm{Cl}\left(\gamma_{+}\right)$is an arc contained in $V_{+}$except one endpoint on $\Delta$ and the other one on $h^{2}(\Delta)$ so it separates $K_{1}$ and $K_{2}$ in $\mathrm{Cl}\left(V_{+}\right)$. This implies $h^{-1}(\Delta) \cap \gamma_{+} \neq \emptyset$. On the other hand, since $\gamma_{+} \subset \mathcal{A}$, by Property 5.8 we get

$$
h^{-1}(\Delta) \cap \gamma_{+}=h^{-1}\left(\Delta \cap h\left(\gamma_{+}\right)\right) \subset h^{-1}\left(\partial_{U} \mathcal{A} \cap \operatorname{Int}(\mathcal{A})\right)=\emptyset,
$$

a contradiction.

We also observe that the two sets $E_{2}$ and $E_{4}$ cannot be simultaneously nonempty since this would give $h^{3}(\Delta) \subset h^{2}\left(V_{+}\right) \cap V_{+}=\emptyset$. It remains to study the situation $h(\Delta) \subset V_{+}$, i.e. $h^{2}(\Delta) \subset h\left(V_{+}\right)$. We first observe that we cannot have $\mathrm{Cl}\left(V_{+}\right) \cup h\left(\mathrm{Cl}\left(V_{+}\right)\right)=\mathbb{S}^{2}$ because this would imply $\Delta \subset h\left(V_{+}\right)$ and then $h(\Delta) \subset h^{2}\left(V_{+}\right) \cap V_{+}=\emptyset$. Thus the whole set $\mathrm{Cl}\left(V_{+}\right) \cup h\left(\mathrm{Cl}\left(V_{+}\right)\right.$is contained in the domain of a single chart of $\mathbb{S}^{2}$. In such a chart, the situation is as in Fig. 10, and $K_{1}$ and $K_{2}$ being fixed points, we obtain a contradiction with the fact that $h$ reverses the orientation. The claim is proved.

We now consider a new "model" homeomorphism $G_{1}$ defined by

$$
\forall(x, y) \in \mathbb{R}^{2} \quad G_{1}(x, y)=(x+|y|,-y) .
$$

Let $D=\left\{(0, y) \in \mathbb{R}^{2} \mid y>0\right\}$ and let $B$ be the domain between $D$ and $G_{1}^{2}(D)$ in the half-plane $P_{+}$. Using again the Schoenflies Theorem, one can construct a homeomorphism $\varphi_{1}: \mathrm{Cl}(B) \rightarrow \mathrm{Cl}\left(V_{+}\right)$such that $\varphi_{1}(0,0)=K_{1}$, 


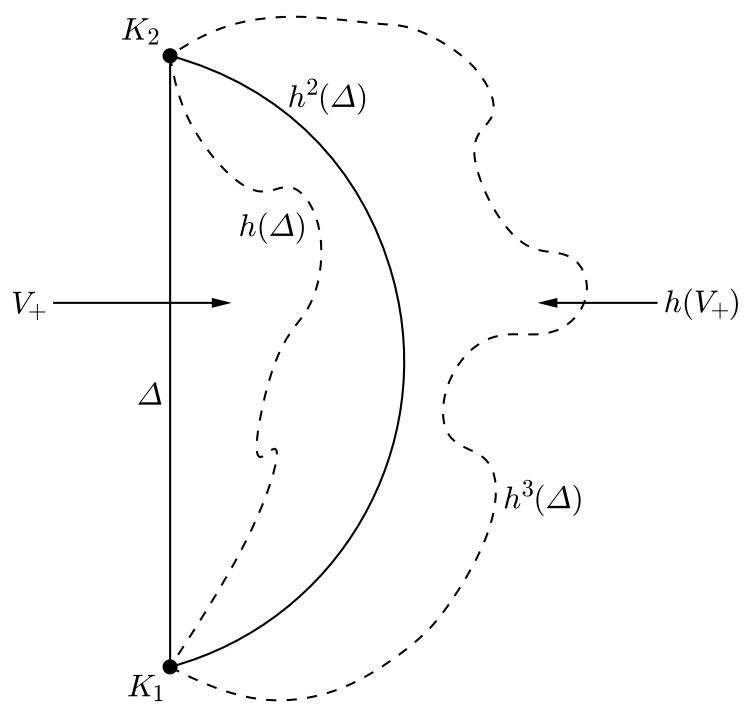

Fig. 10. The situation $h(\Delta) \subset V_{+}$is not possible

$\varphi_{1}(\infty)=K_{2}, \varphi_{1}(D)=\Delta$ and $\left.\varphi_{1} \circ G_{1}^{2}\right|_{D}=\left.h^{2} \circ \varphi_{1}\right|_{D}$. For every $z \in P_{+}$there exists a unique even integer $2 k$ such that $z \in G_{1}^{2 k}(D \cup B)$ and we set

$$
\varphi_{1}(z)=h^{2 k} \circ \varphi_{1} \circ G_{1}^{-2 k}(z) \in h^{2 k}\left(\Delta \cup V_{+}\right) .
$$

In this way $h^{2} \circ \varphi_{1}=\left.\varphi_{1} \circ G_{1}^{2}\right|_{P_{+}}$. Extending $\varphi_{1}$ on $P_{-}$by

$$
\forall y<0 \quad \varphi_{1}(x, y)=h \circ \varphi_{1} \circ G_{1}^{-1}(x, y) \in \bigcup_{k \in \mathbb{Z}} h^{2 k+1}\left(\Delta \cup V_{+}\right),
$$

we obtain a continuous map $\varphi_{1}$ defined on $\mathcal{O}=\left\{(x, y) \in \mathbb{R}^{2} \mid y \neq 0\right\}$ and such that $h \circ \varphi_{1}=\left.\varphi_{1} \circ G_{1}\right|_{\mathcal{O}}$. Using Claim 8 and Lemma 5.2 we get $h^{k}\left(\Delta \cup V_{+}\right) \cap h^{l}\left(\Delta \cup V_{+}\right)=\emptyset$ for $k \neq l$, which ensures that $\varphi_{1}$ is one-to-one. Finally, it is easy to construct a homeomorphism $\psi: \mathcal{O} \rightarrow \mathcal{O}$ such that $G_{1} \circ \psi=\left.\psi \circ G\right|_{\mathcal{O}}$ and

$$
\forall x \in \mathbb{R} \quad \mathrm{Cl}(\psi((\{x\} \times \mathbb{R}) \cap \mathcal{O})) \backslash \psi((\{x\} \times \mathbb{R}) \cap \mathcal{O})=\{(0,0), \infty\} .
$$

Then $\varphi=\varphi_{1} \circ \psi$ is a proper topological embedding such that $h \circ \varphi=\left.\varphi \circ G\right|_{\mathcal{O}}$, with $\varphi(\mathcal{O})=\bigcup_{k \in \mathbb{Z}} h^{k}\left(\Delta \cup V_{+}\right)$. The proof of Theorem 5.1 is complete.

\section{References}

[1] M. Bonino, Lefschetz index for orientation reversing planar homeomorphisms, Proc. Amer. Math. Soc. 130 (2002), 2173-2177.

[2] L. E. J. Brouwer, Beweis des ebenen Translationssatzes, Math. Ann. 72 (1912), $37-54$.

[3] M. Brown, A new proof of Brouwer's lemma on translation arcs, Houston J. Math. 10 (1984), 35-41. 
[4] M. Brown and J. M. Kister, Invariance of complementary domains of a fixed point set, Proc. Amer. Math. Soc. 91 (1984), 503-504.

[5] A. Dold, Lectures on Algebraic Topology, 2nd ed., Springer, Berlin, 1980.

[6] D. B. A. Epstein, Pointwise periodic homeomorphisms, Proc. London Math. Soc. 42 (1981), 415-460.

[7] A. Fathi, An orbit closing proof of Brouwer's lemma on translation arcs, Enseign. Math. 33 (1987), 315-322.

[8] J. Franks, Generalisations of the Poincaré-Birkhoff theorem, Ann. of Math. 128 (1988), 139-151.

[9] -, A new proof of the Brouwer plane translation theorem, Ergodic Theory Dynam. Systems 12 (1992), 217-226.

[10] J. Franks and J. Llibre, Periods of surface homeomorphisms, in: Contemp. Math. 117, Amer. Math. Soc., 1991, 63-77.

[11] G. Graff and P. Nowak-Przygodzki, Fixed point indices of iterations of planar homeomorphisms, preprint.

[12] L. Guillou, Théorème de translation plane de Brouwer et généralisations du théorème de Poincaré-Birkhoff, Topology 33 (1994), 331-351.

[13] —, A simple proof of P. Carter's theorem, Proc. Amer. Math. Soc. 125 (1997), 1555-1559.

[14] B. de Kerékjártó, Topology (I), Springer, Berlin, 1923.

[15] P. Le Calvez, Une version feuilletée du théorème de translation de Brouwer, Comment. Math. Helv. 79 (2004), 229-259.

[16] P. Le Calvez et A. Sauzet, Une démonstration dynamique du théorème de translation de Brouwer, Exposition. Math. 14 (1996), 277-287.

[17] F. Le Roux, Homéomorphismes de surfaces. Théorèmes de la fleur de Leau-Fatou et de la variété stable, Astérisque 292 (2004).

[18] M. H. A. Newman, Elements of the Topology of Plane Sets of Points, Cambridge Univ. Press, 1964.

[19] A. Sauzet, Applications des décompositions libres à l'étude des homéomorphismes de surfaces, thèse, Université Paris 13, 2001.

Institut Galilée, Département de Mathématiques

Université Paris 13

Avenue J.B. Clément

93430 Villetaneuse, France

E-mail: bonino@math.univ-paris13.fr

Received 20 May 2003;

in revised form 2 April 2004 